\title{
Instability modes and transition of pulsatile stenotic flow: pulse-period dependence
}

\author{
H. M. BLACKBUR ${ }^{1}$ AND S. J. SHER WIN ${ }^{2}$ \\ ${ }^{1}$ CSIRO Manufacturing and Infrastructure Technology, PO Box 56, Highett, \\ Vic 3190, Australia \\ ${ }^{2}$ Department of Aeronautics, Imperial College London, South Kensington Campus, \\ London SW7 2AZ, UK
}

(Received 14 October 2005 and in revised form 27 July 2006)

The instability modes arising within simple non-reversing pulsatile flows in a circular tube with a smooth axisymmetric constriction are examined using global Floquet stability analysis and direct numerical simulation. The sectionally averaged pulsatile flow is represented with one harmonic component superimposed on a time-mean flow. We have previously identified a period-doubling global instability mechanism associated with alternating tilting of the vortex rings that are ejected out of the stenosis throat with each pulse. Here we show that while alternating tilting of vortex rings is the primary instability mode for comparatively larger reduced velocities associated with long pulse periods (or low Womersley numbers), for lower reduced velocities that are associated with shorter pulse periods the primary instability typically manifests as azimuthal waves (Widnall instability modes) of low wavenumber that grow on each vortex ring. Convective shear-layer instabilities are also supported by the types of flow considered. To provide an insight into the comparative role of these types of instability, which have still shorter temporal periods, we also introduce high-frequency low-amplitude perturbations to the base flows of the above global instabilities. For the range of parameters considered, we observe that the dominant features of the primary Floquet instability persist, but that the additional presence of the convective instability can have a destabilizing effect, especially for long pulse periods.

\section{Introduction}

Pulsatile flow in a straight tube with a smooth axisymmetric constriction provides an idealization of vascular flow in a stenosed artery, where such occlusions are typically associated with atherosclerotic plaques. The close association between arterial disease and flow-related mechanisms (Caro, Fitz-Gerald \& Schroter 1971) has motivated a number of studies of flow within both idealized and anatomically correct model stenoses, as reviewed by Berger \& Jou (2000).

Some of the earliest investigations into stenotic transitional flows were undertaken experimentally in idealized axisymmetric stenotic tubes (Cassanova \& Giddens 1978; Khalifa \& Giddens 1981; Ahmed \& Giddens 1983, 1984; Ojha, Cobbold, Johnston \& Hummel 1989). The application of computational fluid dynamics has more recently made an impact, through numerical simulations of both idealized and anatomical geometries (Stroud, Berger \& Saloner 2000, 2002; Long et al. 2001; Mallinger \& Drikakis 2002). This body of work has tended to consider either axisymmetric geometries or more complex anatomically derived geometries which cannot be readily reproduced. In contrast, the complementary problem of a two-dimensional 
channel has been investigated in both symmetric and non-symmetric, yet idealized, configurations although even for the symmetric configuration the flow instabilities appear quite distinct from those that arise in axisymmetric geometries (Sobey \& Drazin 1986; Pedley 2000; Mittal, Simmons \& Najjar 2003; Pitt, Sherwin \& Theofilis 2005). Varghese, Frankel \& Fischer 2006 examine flow transition in a non-symmetric idealized constricted tube.

In nearly all of the above studies, flow instability and transition to turbulence has been a common focus. Motivation is provided by the fact that under standard physiological conditions, arterial flows are usually considered to be laminar, although always unsteady and often with separation. However, in the case of a stenotic pipe flow, the increase in local Reynolds number due to the constriction and the introduction of an inflection point into the velocity profile downstream of the constriction can lead to flow transition.

Experimental studies of pulsatile stenotic flows with axisymmetric contractions have suggested that transition occurs a few pipe diameters downstream of the stenosis, does not always persist over the whole pulse cycle, and that flow relaminarizes downstream. For example Ojha et al. (1989), in a flow-visualization experiment with a $75 \%$ occlusion stenosis, divided the post-stenotic flow into four zones. Zone I, reaching to three diameters downstream, is called the 'stable jet region', although some indication of wavy structure is observed on the jet front in this region; Zone II, spanning 3-4.5 diameters downstream, is called the 'transition region', where the waves become larger; in Zone III, the 'turbulent region', 4.5-7.5 diameters downstream, the front rapidly distorts; Zone IV, further downstream again, is labelled 'relaminarization' Khalifa \& Giddens (1981) had drawn attention to shear-layer oscillations that can grow after the passage of each 'starting structure'.

Experimental investigation and/or direct numerical simulation of turbulent flows does not necessarily provide a sound basis for understanding the underlying instability mechanisms leading to transition. Insight into these mechanisms can often be obtained by the linearized stability analysis of a steady or time-periodic flow. For purely oscillatory flows in unconstricted straight tubes, instability mechanisms have been investigated by Yang \& Yih (1977) and Akhavan, Kamm \& Shapiro (1991). We have applied global Floquet analysis of the governing equations, linearized about a twodimensional axisymmetric state which included the stenotic geometry, and coupled this with direct numerical simulation of transitional flows (Sherwin \& Blackburn 2005). That work identified the 'starting structures' as vortex rings and studied the instability modes and transition for both single- and two-harmonic pulsatile stenotic flow in the parameter range of time-averaged Reynolds number (based on mean flow and diameter) between 250 and 550 and non-dimensional pulse period (or equivalently reduced velocity) from 2.5 to 7.5 . It was demonstrated that in the range of parameters investigated, the transition to turbulence was initiated by a period-doubling instability of the vortex rings that are ejected from the stenosis during each pulsatile cycle. The transitional behaviour agrees in broad terms with the zonal categorization supplied by Ojha et al. (1989), although the specifics of the length scales were somewhat different. Another finding of our earlier work was that the extended jet shear layer which rolls up to form vortex rings can be susceptible to convective instability, which is almost certainly the same phenomenon as the shear-layer oscillations observed by Khalifa \& Giddens (1981) and probably accounts for the wavy structures observed in Ojha et al.'s Zone I.

In the present work we return to the same theme, but provide a more systematic and expansive parametric study of the effect of pulse period on instability modes 
(a)

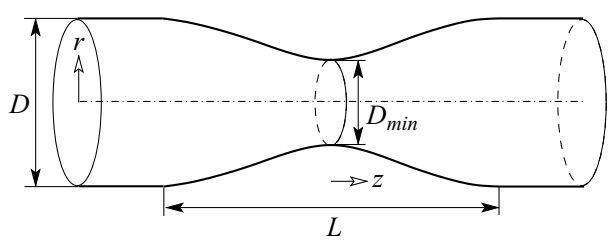

(b)

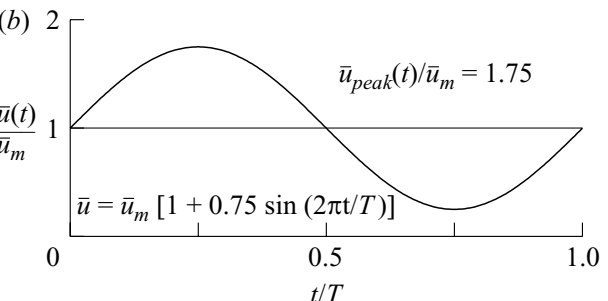

FIgURE 1. (a) Stenosis geometry and (b) pulsatile flow waveform considered in this study.

primarily based around a single-harmonic (mean plus oscillatory) stenotic flow. In expanding the scope of the investigation we have now identified two different global instability mechanisms which can arise in pulsatile stenotic pipe flows: a perioddoubling vortex-tilting instability and a short-wavelength vortex core instability. Both are associated with the creation and transport of a new vortex ring during each pulse, which is a fundamental feature of pulsatile stenotic flow.

However, convective shear-layer instabilities can also be important in these flows. To examine the potential influence of shear-layer instabilities in conjunction with the above global instabilities, we have also considered the case where a high-frequency low-amplitude perturbation is introduced to the base flow. Initially considering the high-frequency perturbation to a steady base flow, we demonstrate that the separated shear layer is susceptible to perturbations with high frequency. This is also true of pulsatile flows, especially those of longer pulse periods, which can have extended shear layers left in the wake of the vortex ring that lies at the leading edge of each pulse. Introducing the most susceptible shear-layer frequency to the pulsatile base flow of the global instabilities indicated that the relatively short pulsatile period vortex core instability is uninfluenced by the perturbation. For longer pulse periods, the period-doubling instability can be significantly enhanced by the perturbations, but the vortex-tilting mechanism remains the same.

\subsection{Problem definition and dimensionless groups}

The stenosis under investigation has a $50 \%$ diametral contraction with a smooth sinusiodal axial profile. Adoption of this level of occlusion $(50 \%$ on diameter, $75 \%$ on area) is motivated by the observation that in clinical practice it is a level readily identified through ultrasound imaging, and it has also been used in a number of other studies. The symmetry of the dynamical system means that a cylindrical coordinate system is the natural choice, and we locate the origin at the centre of the stenosis, as indicated in figure $1(a)$. The axial profile of the stenosis is given by

$$
r(z)=0.5 D_{\text {min }}+0.5\left(D-D_{\text {min }}\right) \sin ^{2}(\pi z / L) \quad \text { over } \quad-0.5 \leqslant(z / L)<0.5,
$$

where $D$ is the tube diameter, $D_{\min }$ is the stenosis throat diameter, and $L$ is its length. Here, $D / D_{\min }=L / D=2$.

In order to quantify the axisymmetric inflow $u(r, t)$ of temporal period $T$, we use the sectional average

$$
\bar{u}(t)=\left(8 / D^{2}\right) \int_{0}^{D / 2} u(r, t) r \mathrm{~d} r
$$

and temporal average

$$
\bar{u}_{m}=\frac{1}{T} \int_{0}^{T} \bar{u}(t) \mathrm{d} t
$$


For given $\bar{u}(t)$ we can define at least three independent dimensionless groups: the reduced velocity $U_{\text {red }}$, the peak-to-mean flow ratio, and the Reynolds number $R e$, respectively given by

$$
U_{\text {red }}=\bar{u}_{m} T / D, \quad U_{p m}=\max _{0 \leqslant t \leqslant T} \bar{u}(t) / \bar{u}_{m}=\bar{u}_{\text {peak }} / \bar{u}_{m}, \quad R e=\bar{u}_{m} D / \nu,
$$

where $v$ is the kinematic viscosity of the fluid. An additional non-independent dimensionless parameter, the Womersley number $\alpha=\left(\pi R e / 2 U_{\text {red }}\right)^{1 / 2}=(2 \pi / T \nu)^{1 / 2} D / 2$, is also commonly used in this applications area, following the application and extension of earlier analysis by Sexl (1930) to work on arterial-type flows in both rigid and elastic tubes by Womersley (1955). We prefer to use the reduced velocity so that the effect of viscosity is parameterized only by Reynolds number, and because the reduced velocity has a simple physical interpretation as the dimensionless distance travelled by the bulk flow in one pulse period. The reduced velocity can also be interpreted as a dimensionless pulse period. The single-harmonic sectional-average flow temporal waveform that is the main focus of the present work is

$$
\bar{u}(t)=\bar{u}_{m}[1+0.75 \sin (2 \pi t / T)],
$$

as illustrated in figure $1(b)$. This is the same as the single-harmonic waveform chosen in Sherwin \& Blackburn (2005), and has a peak-to-mean ratio $U_{p m}=1.75$. (Naturally occurring arterial flows have a broader frequency content and often also have a higher peak-to-mean ratio, although lower values are also observed.) In $\S 6$, we also examine high-frequency convective shear-layer instability both for steady and pulsatile flows, with a much smaller (perturbation-level) oscillation amplitude $\varepsilon=0.001$.

Inlet flows are computed from Womersley's solution for fully developed periodic pulsatile laminar flow in a circular tube, which is the sum of Fourier-Bessel components at each temporal harmonic $n$ defined by

$$
u_{n}(r, t)=\operatorname{Re}\left[\frac{A_{n} \mathrm{i} T}{\rho 2 \pi n}\left(\frac{J_{0}\left(\mathrm{i}^{3 / 2} \alpha 2 r / D\right)}{J_{0}\left(\mathrm{i}^{3 / 2} \alpha\right)}-1\right) \exp 2 \pi \mathrm{i} n t / T\right],
$$

where $\mathrm{i}=(-1)^{1 / 2}, J_{0}$ is the complex Bessel function of zeroth order, $\rho$ is the fluid density, and $\alpha$ is the aforementioned Womersley number. The parameter $A_{n}$ is a complex number representing the driving pressure gradient $\partial_{z} p=A_{n} \exp 2 \pi \mathrm{i} n t / T$ which can be determined from a specified sectional-average velocity $\bar{u}(t)$ at a given harmonic $n$. In the present study, we have primarily restricted consideration to $n \in\{0,1\}$; when $n=0,(1.2)$ reduces to the standard parabolic Hagen-Poiseuille profile for steady flow, $u=2\left(1-4 r^{2} / D^{2}\right)$. In the study of the effect of convective shear-layer instability on pulsatile flows found in $\S 6.2$, we extend the set of harmonics to include an additional high frequency.

As is commonly justified in many works on blood flows in the major arteries, Newtonian rheology has been assumed. Although the effect of artery wall compliance is potentially important, it has been ignored in the present work on the assumption that the major role of the compliance - a distributed property - will be to influence the shape of the waveform reaching a given site, rather than to significantly affect local flow dynamics. For discussion of these assumptions and reviews on blood flow in arteries, see Ku (1997), Wootton \& Ku (1999), Berger \& Jou (2000) and Taylor \& Draney (2004).

\subsection{Outline of paper}

In $\S 2$, the numerical and analytical methods employed are described in brief. In order to provide context for the stability analysis to follow, the effect of variation 
in reduced velocity (pulse period) on base flows at fixed $R e=400$ is examined in $\S 3$. In $\S 4$, we use Floquet stability analysis and three-dimensional direct numerical simulation (DNS) to study the effect of $U_{\text {red }}$ on the period-doubling mode: this is a superset of the three single-harmonic results presented in our earlier paper. We also introduce phase-averaged statistics to help quantify the corresponding effect of $U_{\text {red }}$ on time-asymptotic turbulent flows following transition; the locations of turbulent breakdowns may evolve to locations considerably upstream of the location of the peak Floquet mode amplitude. In $\S 5$, we show that at lower reduced velocities (shorter pulse periods) than for the period-doubling mode, the primary instability manifests as azimuthal waves, evocative of the Widnall modes observed for isolated vortex rings (Widnall, Bliss \& Tsai 1974), and again we also study the flow following transition using three-dimensional DNS. At still shorter periods, the shear layers of steady and long, pulse period stenotic flows are found to be susceptible to a convective instability, which we examine by adding a harmonic perturbation to the inflow in axisymmetric DNS and following this through to the effects on Floquet instability and three-dimensional DNS; these aspects are dealt with in $\S 6$. The whole study is summarized and discussed in $\$ \S 7$ and 8 .

\section{Numerical methods}

For complete details of the numerical methods and their prior application to this problem, consult Blackburn (2002), Blackburn \& Sherwin (2004) and Sherwin \& Blackburn (2005). In the following, we summarize the numerical and analytical techniques employed.

The paper addresses the linear Floquet stability analysis and direct numerical simulation of the incompressible Navier-Stokes equations, which are treated in cylindrical coordinates $(z, r, \theta)$. The spatial discretization couples spectral elements having nodal Gauss-Lobatto-Legendre (GLL) basis functions in the meridional semiplane with Fourier basis functions in the azimuth. Axisymmetric flows can be considered as the zeroth azimuthal Fourier mode in such a scheme. Temporal integration is performed using a mixed implicit-explicit pseudo-spectral velocity correction scheme of second order (Karniadakis, Israeli \& Orszag 1991; Guermond \& Shen 2003).

The spectral element macro mesh employed both in the present study and also in the single-harmonic part of Sherwin \& Blackburn (2005) is illustrated in figure 2. There are 743 spectral elements, concentrated around the stenosis throat and where the shear layers are thinnest. The comparatively long outflow was found necessary to accommodate and resolve the Floquet modes, which can reach their greatest amplitude some distance downstream of the stenosis. In the previous study, a GLL basis function order $N_{p}=7$ was found adequate to resolve Floquet multipliers to approximately four-figure accuracy, and has been retained through the present work. The mesh has approximately 38000 independent degrees of freedom for each azimuthal semi-plane, i.e. a semi-plane defined by a fixed angle $\theta$ and $r \geqslant 0$.

Temporal Floquet analysis examines the behaviour of a perturbation $\boldsymbol{u}^{\prime}$ to a $T$ periodic base flow $\boldsymbol{U}$, to determine whether the perturbation grows or decays from cycle to cycle. In a linear analysis, the evolution equations for the perturbation flow are the Navier-Stokes equations linearized about the base flow. Perturbation solutions can be written as a sum of Floquet modes $\tilde{\boldsymbol{u}}\left(t_{0}\right) \exp \sigma\left(t-t_{0}\right)$ where $\tilde{\boldsymbol{u}}\left(t_{0}\right)$ is a $T$-periodic Floquet eigenfunction, evaluated at arbitrary phase $t_{0}$, and $\sigma$ is a Floquet exponent. Floquet multipliers $\mu$ are related to the Floquet exponents by $\mu=\exp \sigma T$, 
(a)
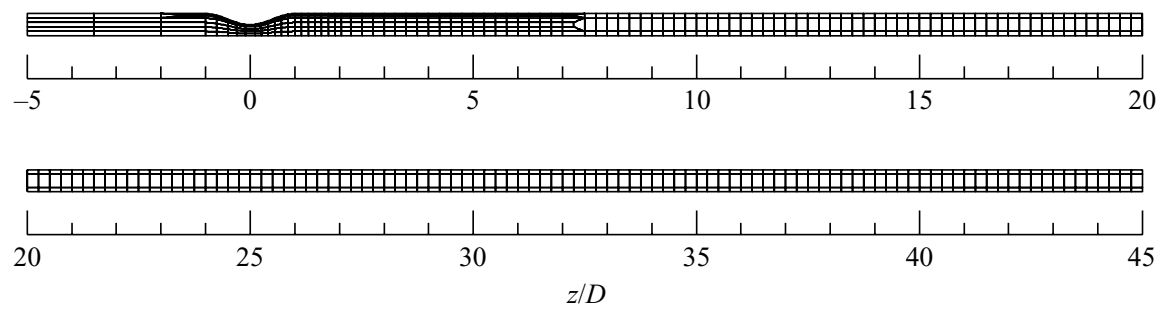

(b)

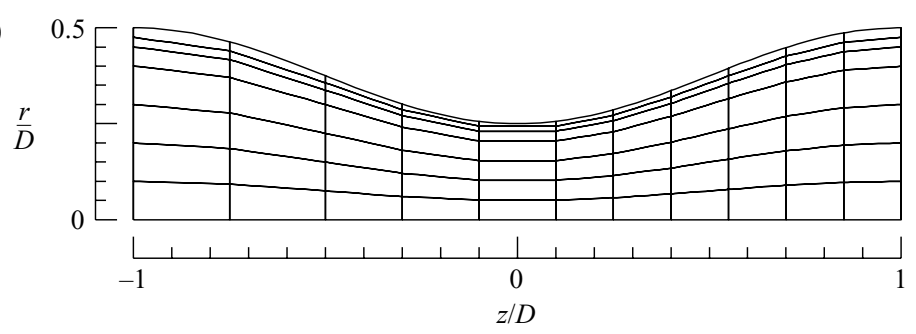

FIgURE 2. Computational mesh in the meridional semiplane. (a) Layout of the 743 spectral elements. (b) Close up of the stenosis.

and indicate how much the Floquet modes grow (or shrink) from cycle to cycle. In general, the exponents, the multipliers, and the eigenfunctions can be real or occur in complex-conjugate pairs. Instability occurs when a multiplier leaves the unit circle, $|\mu|=1$, or equivalently when the real part of a Floquet exponent becomes positive. In the present treatment, Floquet modes can take any spatial form supported in a fixed frame of reference by the global $(r, z)$ discretization and hence, by definition, an unstable Floquet mode is a global instability.

As noted by Barkley \& Henderson (1996) in the case (as here) where base flows are two-dimensional two-component, we may employ restricted-symmetry mode shapes of type

$$
(\tilde{u}, \tilde{v}, \tilde{w})(z, r, \theta, t)=(\hat{u} \cos k \theta, \hat{v} \cos k \theta, \hat{w} \sin k \theta)(z, r, t),
$$

where $(\tilde{u}, \tilde{v}, \tilde{w})$ are, respectively, axial, radial and azimuthal velocity components, and with $k$ an integer wavenumber. Since for the restricted class of base flows the above azimuthal structure is invariant under the linearized Navier-Stokes operator, expansions described by (2.1) are suitable candidates for eigenmodes. This is true without further qualification if the Floquet multipliers are real. If multipliers are, however, found to be complex-conjugate, then, as explained by Blackburn \& Lopez (2003), an expanded set of basis functions should also be used to compute (modulated) travelling-wave modes, while (2.1) gives a (modulated) standing wave. If the multiplier is real, or if one is only considering the modulated standing wave in the complexconjugate case, then the above expansion provides an instance of the complete set of instability modes that forms a pitchfork of revolution in which the non-axisymmetric mode shapes can be arbitrarily rotated about the cylindrical axis. As was the case in Sherwin \& Blackburn (2005), all the unstable Floquet modes in the present investigation are found to occur with real multipliers, so (2.1) has been used for all Floquet analysis cases presented, whereas for DNS, complete (i.e. fully complex) Fourier bases were adopted.

The following boundary conditions were applied. At the inflow, velocities were computed from (1.2) and imposed at $z / D=-5$ when computing either 


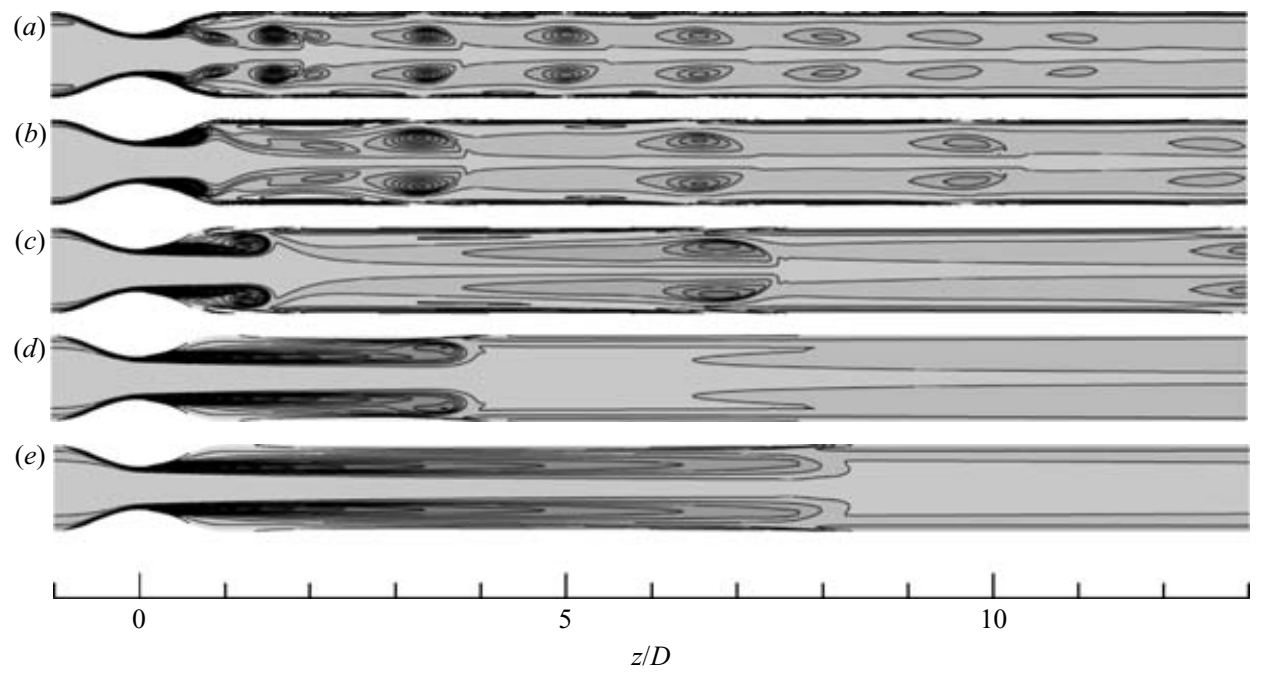

FIGURE 3. The effect of pulse period on base flow vorticity on a section through the tube centreline at $R e=400$. (a) $U_{\text {red }}=0.5, \alpha=33.45 ;$ (b) $U_{\text {red }}=1, \alpha=25.07 ;$ (c) $U_{\text {red }}=2$, $\alpha=17.72 ;(d) U_{\text {red }}=5, \alpha=11.21 ;(e) U_{\text {red }}=10, \alpha=7.93$. Fifteen contour levels equispaced between $\omega_{\theta}=-10 \bar{u}_{m} / D$ and $100 \bar{u}_{m} / D$, at phase $t=j T, j \in \mathbb{I}$.

two-dimensional DNS (e.g. for base flows) or three-dimensional DNS. Outflow boundary conditions of type $(\partial \boldsymbol{u} / \partial z=0, p=0)$ are used in both DNS and stability analysis at $z / D=45$. All other boundary conditions (including at the inflow boundary when carrying out stability analysis) are of wall type, i.e. $\boldsymbol{u}=0$, and with a pressure boundary condition of computed Neumann type (Karniadakis et al. 1991; Blackburn \& Sherwin 2004).

Stability analysis is computed using an explicitly restarted Arnoldi method described by Barkley \& Henderson (1996), Tuckerman \& Barkley (2000) and Blackburn (2002). Typically, these methods rely on the repeated application of an operator (here, the linearized Navier-Stokes operator, integrated over a period $T$ ), rather than explicit computation of the equivalent matrix operator, and deliver some small number of leading modes. While checking the computations presented herein, we made a detailed study of the influence of the Krylov dimension on the computed mode shape. We found that while the computed Floquet multiplier was robust, the mode shape could become corrupt if the Krylov dimension was made too large (here, more than about 20 ), and for all the computations presented, the Krylov dimension was either 8 or 12 . The corruption is most probably related to finite-precision limitations in a modified Gram-Schmidt vector orthogonalization that forms part of the iteration.

\section{Axisymmetric base flows}

The purpose of this section is to introduce the main characteristics of the singleharmonic base flows used in subsequent stability analysis. We temporarily restrict attention to a single Reynolds number, $R e=400$.

The primary visual feature of pulsatile stenotic flow is vortex rings that form downstream of the stenosis from roll-ups of the shear layer that separates in the throat, just past the location of minimum cross-section. When only a single harmonic is present, a single ring forms with each pulse period. Figure 3 shows the effect of reduced velocity (dimensionless pulse period) on the vorticity contours of the 


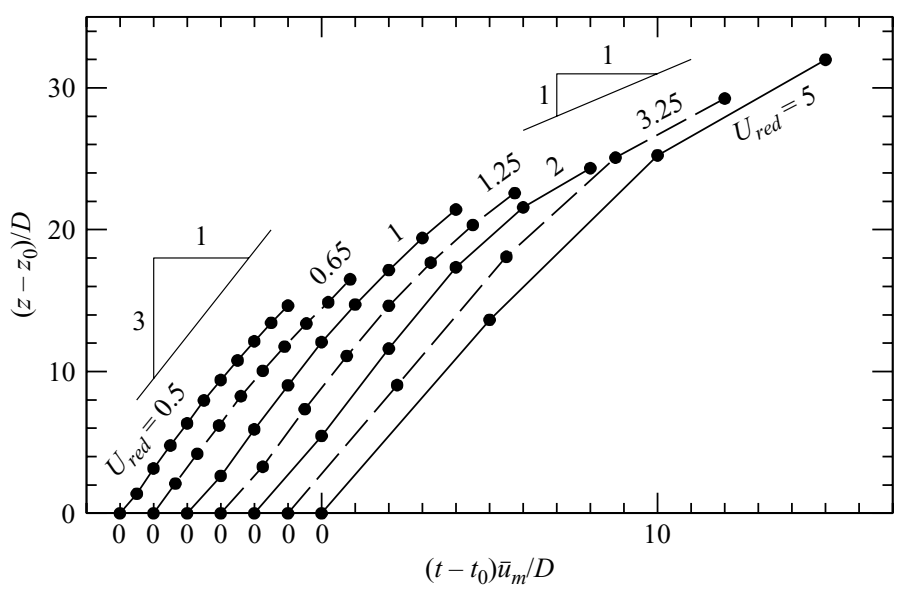

FIGURE 4. Dimensionless axial locations of base flow vortex rings at $t=j T$, as a function of dimensionless time (ring formation pulse index $\times U_{\text {red }}$ ) at various values of $U_{\text {red }}=\bar{u}_{m} T / D$ for $R e=400$.

axisymmetric flows. In this plot, all simulations are at phase point $t=j T, j \in \mathbb{I}$. As may be expected, the effect of increasing $U_{\text {red }}$ is to increase the axial spacing of successive vortex rings. As $U_{\text {red }}$ increases, elongated shear layers may be observed in the wakes of the primary shear-layer roll-ups. We shall later find that the dynamics of these shear layers can contribute significantly to the overall flow behaviour. For the lower reduced velocities (figure $3 a, b$ ) there is also some indication of secondary ring structures appearing near the first fully separated vortex ring. As the vortex rings move further downstream, their vorticity diffuses away and they eventually disappear; this is especially evident in figure $3(a)$.

A feature that is apparent from a comparison of vortex spacings in figure $3(a-c)$, where the reduced velocities increase by successive factors of two, is that the initial traverse speed of the vortex rings is approximately constant, independent of $U_{\text {red }}$. To investigate this point in more detail, we show in figure 4 the axial locations of successive identifiable vortex rings (taken as those of the local maxima of vorticity) relative to the location, $z_{0}$, of the first identifiable vortex ring, as a function of time from an arbitrary phase point, $t_{0}$. The values were obtained from instantaneous snapshots of the flow taken at the same phase point, $t=j T$, for each value of $U_{\text {red }}$. For any value of $U_{\text {red }}$, the task of identifying individual vortices was abandoned when there were no further readily discernable maxima of vorticity along an axial traverse. It may be seen that for all reduced velocities, the intial vortex ring speed is approximately the same, with an approximate value $3 \bar{u}_{m}$. We note that the ring translation speeds are considerably less than the peak bulk velocity at the thoat, which is $U_{p m}\left(D / D_{\min }\right)^{2} \bar{u}_{m}=7 \bar{u}_{m}$, and lower also than the mean bulk velocity at the throat, $\left(D / D_{\min }\right)^{2} \bar{u}_{m}=4 \bar{u}_{m}$. That the initial translation speeds are approximately independent of $U_{\text {red }}$ has a one-to-one correspondence with the fact that the initial spacing of successive rings scales approximately linearly with $U_{\text {red }}$, as was observed in relation to figure 3 : the spacing is $\Delta z / D \approx 3 U_{\text {red }}$.

Sufficiently far downstream of the stenosis, and with laminar flow, one may expect the velocity profile to recover to the upstream value provided by (1.2). As the strength of an individual vortex falls to zero, relative to that of the inflow profile, it is reasonable to assume that its average advection velocity falls to approximately $\bar{u}_{m}$. 
This behaviour may be noted in figure 4, for the higher values of $U_{\text {red }}$ and at large times. On the other hand, for small values of $U_{\text {red }}$, the viscous decay of vortices completes well before the flow recovers to the inlet profile, and so the asymptotic speeds appear much higher.

The initial vortex ring speeds are approximately independent of reduced velocity, which implies that the circulations around the vortex cores are approximately independent of pulse period. On the other hand, the pulse-integral vorticity flux from the separation zone must increase approximately linearly with reduced velocity, since although the bulk velocities are independent of $U_{\text {red }}$, this quantity is proportional to the period $T$. The behaviour is apparently related to what is observed for isolated laminar vortex rings generated by motion of a piston in a tube, ejecting into a large body of fluid (Gharib, Rambod \& Shariff 1998; Rosenfeld, Rambod \& Gharib 1998). As piston stroke increases, normalized circulation (and hence speed) of the rings increases too, but only to a universal limiting value known as a 'formation number'. At higher stroke levels, excess vorticity is pinched off from the vortex ring, and trails behind it as a shear layer (which may, in turn, roll up into a subsidiary train of discrete vortices). Similar behaviour can be observed for the higher reduced velocity flows of figure $3(d, e)$, where elongated shear layers trail behind rolled-up vortex rings.

A final point to be made in relation to figure 4 is that, at the same Reynolds number, vortex rings survive as identifiable entities for longer times as reduced velocity (or dimensionless pulse period) is increased.

\section{Period doubling instability mode}

Axisymmetric time-periodic base flows are the state about which we linearize prior to conducting Floquet analysis for three-dimensional instabilities. At any reduced velocity, base flows are pre-computed for a range of Reynolds number, and the dominant Floquet multiplier $\mu$ and its associated eigenfunction (at a specific phase point $t / T)$ is established at each Reynolds number. By interpolating to $|\mu|=1$, the marginal stability Reynolds number is established, as shown in figure 14 of Sherwin \& Blackburn (2005). All the pulsatile flow instability modes discovered in that study had $\mu_{c}=-1$, which corresponds to a period-doubling bifurcation. The instability can be physically associated with a tilting of the vortex rings, and the period-doubling mechanism was shown to be that the downwash induced by this tilting in the wake of one ring drives an opposite tilt on the succeeding ring. Because the mechanism of this instability mode was described in some detail in Sherwin \& Blackburn (2005), the treatment of this aspect here will be somewhat abbreviated, and concentrate more heavily on the effects of variation of pulse period.

\subsection{Linear stability}

The tilting mechanism necessarily arises in the first azimuthal Fourier mode, wavenumber $k=1$. In figure 5, we show an example wavenumber spectrum of Floquet multiplier magnitudes computed at $U_{\text {red }}=3.25, R e=400$, which shows all the other modes to be stable, with the least stable of these occurring at $k=4$.

Through the process of interpolation at each reduced velocity, we have established the curve of marginal stability of the period-doubling mode as a function of $U_{\text {red }}$, shown in figure 6. An 'optimal' reduced velocity (minimum $R e_{c}$ ) occurs near $U_{\text {red }}=$ 3.25, where $R e_{c}=380$. At $U_{\text {red }}<2, R e_{c}$ for this mode grows rapidly to high values, while it appears that in the high $U_{\text {red }}$ limit, $R e_{c}$ grows approximately linearly with $U_{\text {red }}$. 


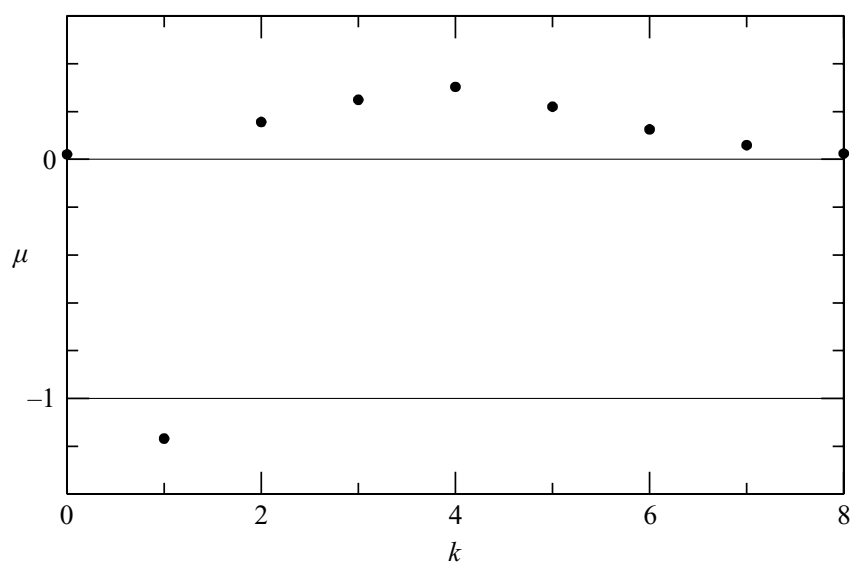

FIGURE 5. Azimuthal wavenumber spectrum of Floquet multipliers at $U_{\text {red }}=3.25, R e=400$. The negative multiplier of the unstable mode at $k=1$ indicates a period-doubling instability.

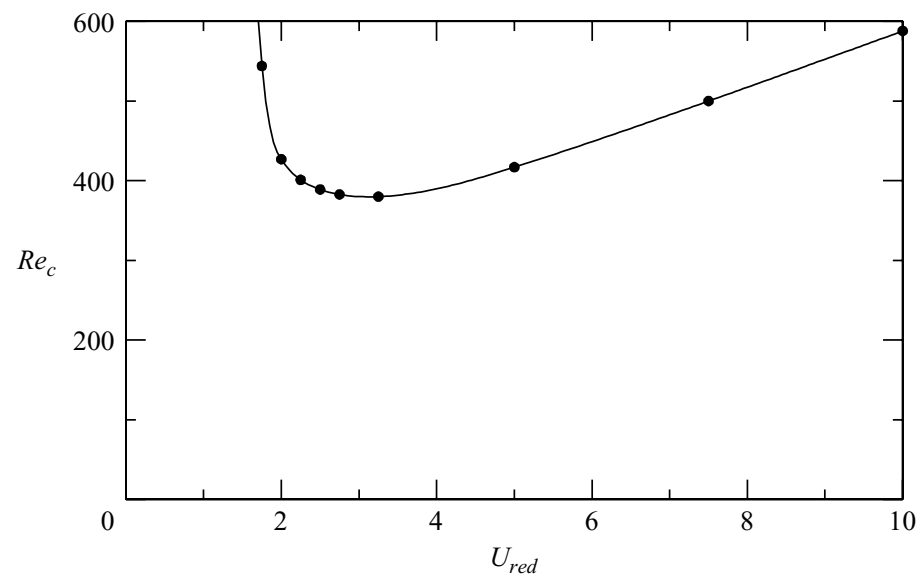

FIGURE 6. Curve of critical Reynolds number for the period-doubling instability mode as a function of reduced velocity.

Figure 7 provides a number of contour plot pairs of instantaneous energy per unit mass $q=\boldsymbol{u}^{2} / 2$ in the base flow and also the logarithm of $q$ for the corresponding period-doubling mode, both illustrated at the phase point $t=j T$ for various $U_{\text {red }}$ and at $R e$ close to $R e_{c}$. The pair (figure $7 e, f$ ) at $U_{\text {red }}=3.25, R e=370$ corresponds to the optimum reduced velocity in figure 6 . The pulsatility of the base flows is readily observed: each local maximum of $q$ here corresponds to a vortex ring. It can be seen that as the optimum reduced velocity, $U_{\text {red }}=3.25$, is approached from below, the peak energy in the Floquet modes migrates upstream towards the stenosis and is associated with sets of vortex rings located progressively closer to the stenosis. However, in all these cases, as a downstream traverse is made from one base flow vortex to the next, the Floquet modal energy first grows, then dies away. We take this to indicate a competition between an instability that tries to grow with distance downstream (or time), while the energy of the base flow pulses, that feed the instability, dissipates. Note that at low reduced velocities, there is significant overlap of contours of modal energy associated with successive base flow pulses, indicative of a strong cooperative 
(a)

(b)

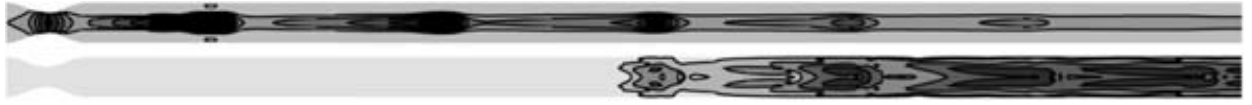

(c)

(d)

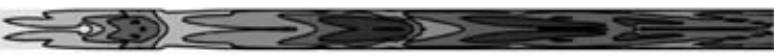

(e)

(f) 8

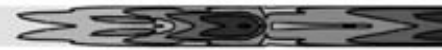

(g)

(h)
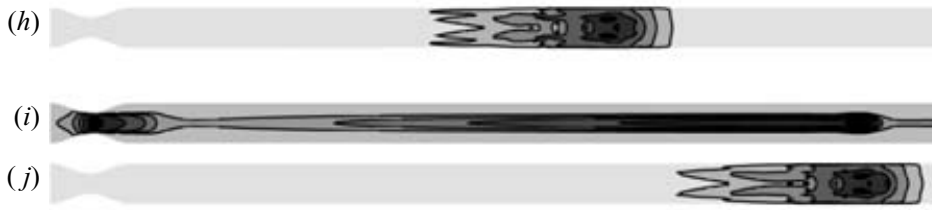

$(k)$

(l)

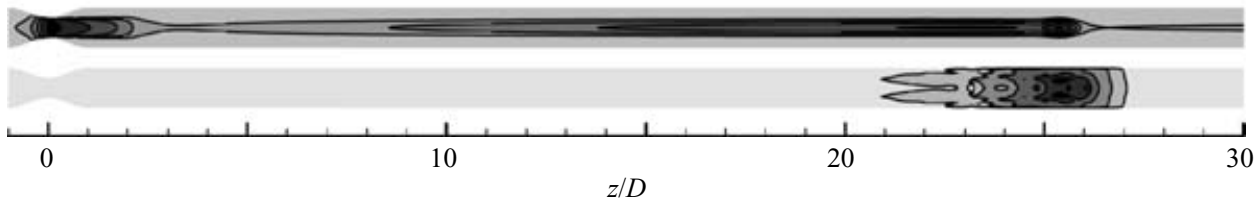

FIGURE 7. Contour plot pairs showing instantaneous energy $q$ in the base flow (top of pair) and logarithm of energy in the period-doubling Floquet instability mode (bottom of pair) on a plane containing the tube centreline for: $(a, b) U_{\text {red }}=1.75, \operatorname{Re}=545 ;(c, d) U_{\text {red }}=2.5$, $R e=389 ;(e, f) U_{\text {red }}=3.25, R e=370 ;(g, h) U_{\text {red }}=5, R e=415 ;(i, j) U_{\text {red }}=7.5, R e=500$; $(k, l) U_{\text {red }}=10, R e=590$. Results are all close to marginal stability and shown at phase point $t=j T$. Base flow contour levels are drawn at $2 \bar{u}_{m}^{2}, 4 \bar{u}_{m}^{2}, \ldots, 10 \bar{u}_{m}^{2}$. Eigenmodes were pre-normalized such that the (two-dimensional) domain-integral of modal kinetic energy is equal to $\bar{u}_{m}^{2} D^{2}$, and has (log) energy contours drawn at decade-and-a-half intervals from -9 to -3 . Note that the visualized outflow region is $15 \mathrm{D}$ shorter than the computational domain.

coupling from the instability on one ring to those on its immediate partners through the alternating-wake-downwash mechanism mentioned above.

At $U_{\text {red }}=3.25$, modal energy contours can just be observed starting to appear on the first base flow pulse downstream of the stenosis, at $z / D \approx 8$. At higher reduced velocities, we observe that the Floquet mode's energy is associated with just the first vortex downstream of the stenosis; this is confirmed by the pair $(g, h)$ where there is no observable modal energy on the second visible base flow pulse downstream of the stenosis. This indicates a reduction in the cooperative coupling between the instability that grows on successive rings, probably brought about by increased axial spacing. We associate the asymptotic high- $U_{\text {red }}$ behaviour seen on the critical curve in figure 6 (where $R e_{c}$ grows linearly with $U_{\text {red }}$ ) with this change in spatial structure of the Floquet energy with respect to the base flow vortex ring locations. We will return to this discussion in $\S 7.2$.

\subsection{Nonlinear asymptotic states}

In DNS studies, we initiate simulations by adding a small component of the leading Floquet mode to the axisymmetric base flow, and projecting the combination to a 
three-dimensional space with a large number of Fourier modes. The simulation is then integrated forward in time until an asymptotic statistically stationary state is reached. As explained in Sherwin \& Blackburn (2005), this evolution may take the order of hundreds of pulse periods to achieve. The typical behaviour is for the instability to gain an initial saturation relatively quickly, with nonlinear behaviour that could be modelled using a low-order normal form. During this phase, the maximum threedimensional energy arises in approximately the same axial location as for the Floquet mode. Subsequently, however, there is a long second saturation to the asymptotic state, in which the peak three-dimensional energy evolves slowly upstream towards the stenosis. As this happens the amount of three-dimensional energy in the flow also increases, associated with the flow making a localized transition to turbulence, but also with the fact that the energy of the vortex rings that feed the instability is greater upstream. In the asymptotic state, the transition typically appears on the vortex ring most recently blown out of the stenosis, even though the peak energy of the Floquet mode may have arisen far downstream.

Figure 8 illustrates the asymptotic state for $U_{\text {red }}=2.5, R e=400$ at phases $t=j T$ and $t=(j+1) T$, i.e. one pulse period apart. See Sherwin \& Blackburn (2005) for details of the progression to this asymptotic state. Three instantaneous isosurfaces are used. An isosurface drawn on a positive value of the discriminant $\mathscr{D}$ of the velocity gradient tensor is used in order to pick out the pulse-driven vortex rings. (As explained by Chong, Perry \& Cantwell (1990), where the discriminant is positive, the velocity gradient tensor has a complex-conjugate pair of eigenvalues, and the flow has a local spiralling character. As demonstrated by Blackburn, Mansour \& Cantwell (1996), isosurfaces based on this measure serve well as markers for isolated vortices, particularly in the presence of walls. In the present context, such isosurfaces are preferable to those of enstrophy, because this quantity takes local maxima on the walls of the tube and hence its isosurfaces tend to obscure vortex structure within.) The other two isosurfaces in figure 8 are drawn on equal-magnitude positive and negative values of axial vorticity component; these emphasize the departure of the flow from axisymmetry.

In figure $8(a)$, there is an isolated, completely axisymmetric isosurface of $\mathscr{D}$ located in the stenosis throat, while approximately $2.5 \mathrm{D}$ downstream, an isolated vortex ring may be seen, tilting streamwise at the top of the tube, and is accompanied near the centreline by two isosurfaces of the streamwise vorticity component as it begins to distort three-dimensionally. Approximately one further diameter downstream, there is a tangle of interlocked isosurfaces that marks a weakly turbulent breakdown of two previous vortex rings. Note also that the dominant tilt at the upstream end of this tangle seems to be the reverse of that for the approaching vortex ring. The breakdown dissipates further downstream, however, and by approximately $8 D$ downstream, relaminarization appears to be well underway. Eventually, the laminar pulsatile flow profile of the inlet flow is regained, although we have not studied the approach to this state in detail.

Now comparing figure $8(b)$ to figure $8(a)$, the period-doubling nature of the instability is readily seen, since the second image is nearly an exact reflection about a horizontal plane of the first. This is true even at the upstream end of the apparently turbulent burst, and the effect is still quite strong near its downstream limit. This feature persists in the statistics when the solution is perturbed through the addition of low-energy random noise.

All the period-doubling instabilities that we have investigated via DNS have shown broadly similar behaviour, in that (i) there is a slow migration of the breakdown 
(a)

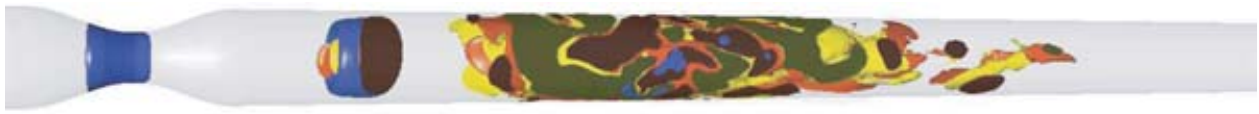

(b)

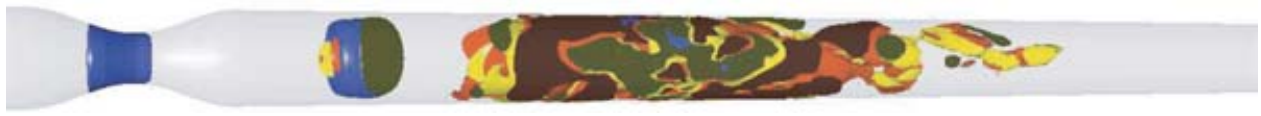

FIGURE 8. Two visualizations of the asymptotic turbulent state for $U_{\text {red }}=2.5, R e=400$, at a time interval $\Delta t=T$. Instantaneous isosurfaces of velocity gradient tensor discriminant (blue, $\mathscr{D}=8 \times 10^{4} \bar{u}_{m}^{6} / D^{6}$ ), and equal magnitudes of positive and negative axial vorticity component (red/yellow, $\left.\omega_{z}= \pm 2 \bar{u}_{m} / D\right)$. In each view, the first well-organized set of structures downstream of the throat represents a vortex ring that has begun to tilt and distort; the second less-organized set results from the breakdown of the two preceding vortex rings. Note that the flow at times $(a)$ and $(b)$ forms almost exact mirror images, consistent with the underlying period-doubling instability.

(a)

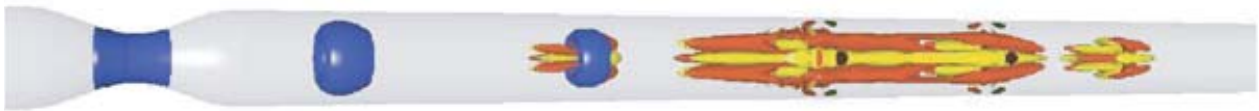

(b)

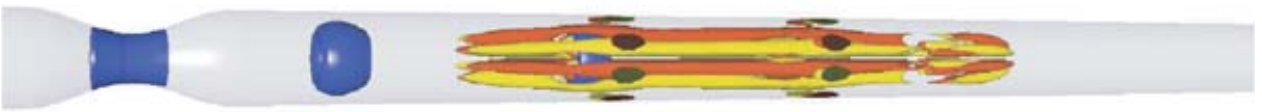

FIGURE 12. Isosurfaces illustrating the $(a)$ wavenumber $k=3$ and $(b)$ wavenumber $k=4$ wavy instability modes at $U_{\text {red }}=0.875, R e=325$, at phase point $t=j T$. Vortex rings of the base flow are visualized on a positive value of the velocity gradient tensor discriminant (blue, $\mathscr{D}=8 \times$ $10^{4} \bar{u}_{m}^{6} / D^{6}$ ), while the wavy mode shapes are visualized using equal-magnitude but oppositely signed values of axial vorticity component (red/yellow, arbitrary equal-magnitude levels).

(a)

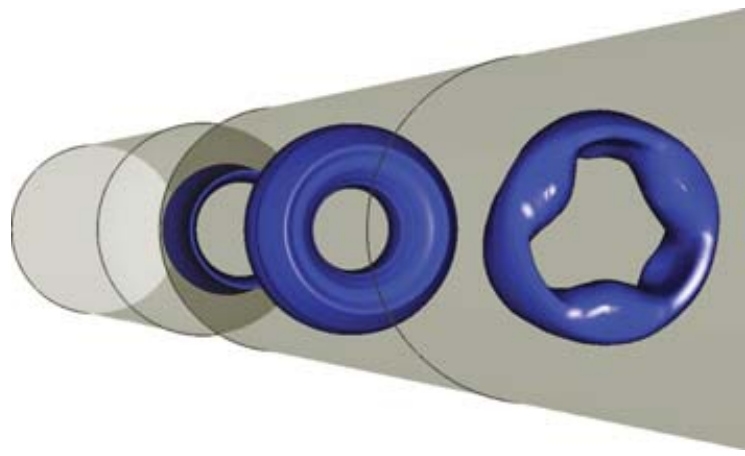

(b)

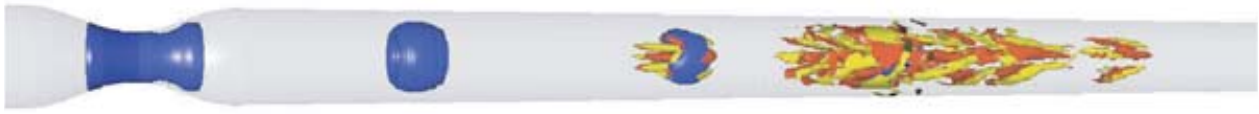

(c)

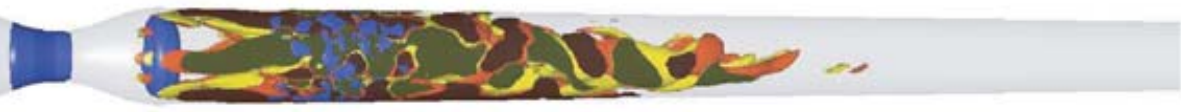

FIGURE 15. Visualizations of turbulent states for $U_{\text {red }}=1, R e=350$, promoted by a $k=3$ instability. (a) The progression to a wavy vortex ring, visualized via an isosurface of velocity gradient discriminant $\mathscr{D}=4 \times 10^{5} \bar{u}_{m}^{6} / D^{6}$, looking upstream to the throat and inlet, for a state after the instability has completed an initial nonlinear saturation. (b) A different view at the same stage in the transition process. Blue-red-yellow isosurfaces as described in caption for figure 8. At stage $(c)$, a number of pulse cycles later, the flow has reached an asymptotic state and turbulent breakdown occurs just a few tube diameters downstream of the throat. 
point upstream to an asymptotic location; (ii) the breakdown is followed by apparent relaminarization downstream, most probably leading to recovery of the far-upstream laminar-pulsatile velocity profile; and (iii) the period-doubling nature of the instability is retained in the nonlinear asymptotic state. As was remarked in $\S 6.4 .4$ of Sherwin \& Blackburn (2005), the overall description of behaviour seen here is in many ways similar to the experimental flow visualization observations made by Ojha et al. (1989) at $R e=575, U_{p m}=1.6$ and $U_{\text {red }}=16.1$ in a tube with a sharp-edged 2:1 diametral contraction. They did not report period-doubling behaviour, but were perhaps unaware of this possibility. We note that no existing study analyses the stability of pulsatile flow in a straight tube, thus it is unclear if we may expect the flow to relaminarize in all cases.

Next, we examine the phase-averaged flows in the asymptotic states for cases $\left(U_{\text {red }}=2.5, R e=400\right),\left(U_{\text {red }}=5, R e=430\right),\left(U_{\text {red }}=7.5, R e=510\right)$ and $\left(U_{\text {red }}=10\right.$, $R e=600$ ). By inspection of figure 6 the Reynolds numbers will be seen to be just slightly greater than the critical values at each reduced velocity. The number of azimuthal Fourier modes (half the number of azimuthal planes of data) was set to 16, 24, 24 and 32, respectively, producing approximately 1.2, 1.8, 1.8 and 2.4 million grid points for the four cases, in recognition of the increasing Reynolds numbers. It has been verified that the spread of Fourier-mode- $k$ kinetic energies

$$
E_{k}=\frac{1}{2 A \bar{u}_{m}^{2}} \int_{\Omega} \hat{\boldsymbol{u}}_{k} \cdot \hat{\boldsymbol{u}}_{k}^{*} r \mathrm{~d} \Omega
$$

(where $\Omega$ is the extent of the two-dimensional meridional semiplane and $\hat{\boldsymbol{u}}_{k}^{*}$ denotes the complex conjugate of the velocity data in the $k$ th Fourier mode) from the first to the last non-axisymmetric mode in each case spans at least three decades, which is thought to be sufficient, given the resolution in the meridional semiplane. The quantity then examined is the turbulent energy $q^{\prime}=\boldsymbol{u}^{\prime 2} / 2$ after the mean and periodic flows have been removed (Reynolds \& Hussain 1972). Considering that only a single contribution to a phase average can be accumulated in each pulse period, we have restricted these calculations to averages over 50 periods; as this is an even number, any alternation associated directly with period-doubling is averaged out. For each flow, we have computed eight phase averages at $t_{i}=(j+i / 8) T$, then their azimuthal averages, and finally, extracted contours of $q^{\prime}$. Outcomes are presented as two-dimensional contour plots on a plane containing the tube centreline; in order to save space we have presented only four of the eight phases collected. Note that contour levels are identical in each case.

Figure $9(a)$ shows the set of contours for phase-averaged turbulent energy computed in this manner for $\left(U_{r e d}=2.5, R e=400\right)$, i.e. corresponding to the state illustrated in figure 8 . There is evidently some spatial structure to the random fluctuations, in agreement with the qualitative remarks made above in relation to figure 8 . We see that the turbulent energy is effectively contained within an approximately $4 D$-long region commencing at $z / D \approx 2.5$. Note the contrast with the linear Floquet mode seen for the same reduced velocity in figure $7(d)$, where the greatest modal energy arises near $z / D=20$.

Figures $9(b)-9(d)$ show the phase-average outcomes in the asymptotic states at $\left(U_{\text {red }}=5, R e=430\right),\left(U_{\text {red }}=7.5, R e=510\right)$ and $\left(U_{\text {red }}=10, R e=600\right)$, respectively. A similar to that pattern of behaviour seen in figure $9(a)$ is observed, but the location of the turbulent burst moves downstream as $U_{\text {red }}$, or equivalently dimensionless pulse period, increases. The axial spread of the energy across a pulse period remains 
(a)

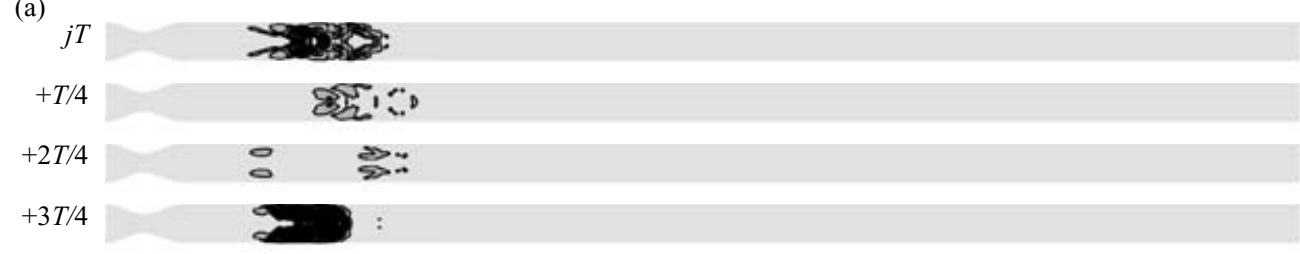

(b)

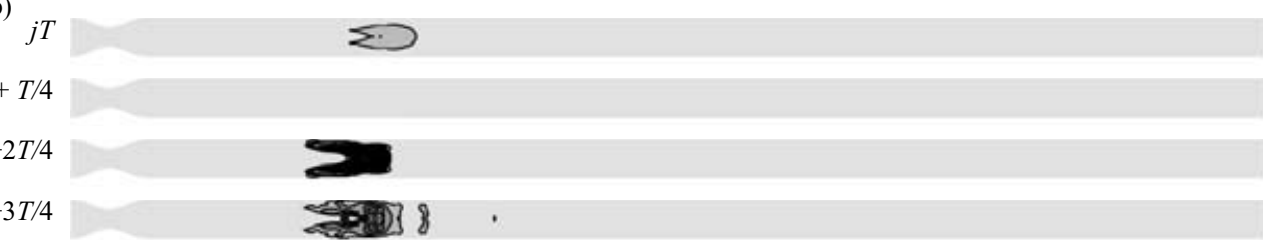

(c) $j T=0$

$+T / 4$

$+2 T / 4$

$+3 T / 4$

S.?

(d)

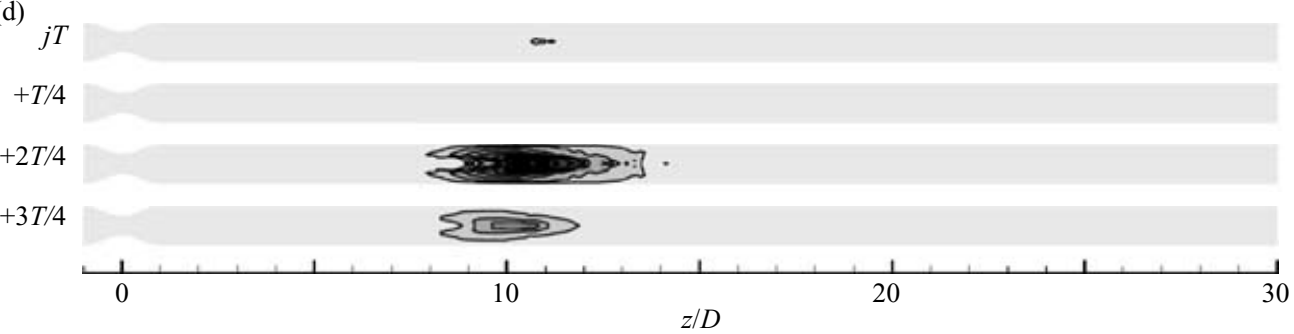

FIGURE 9. Contours of phase-averaged turbulent energy on a slice through the pipe centreline at four temporal phases for the asymptotic states at $(a) U_{\text {red }}=2.5, R e=400 ;(b) U_{\text {red }}=5$, $R e=430 ;(c) U_{\text {red }}=7.5, R e=510 ;(d) U_{\text {red }}=10, R e=600$. The contour levels of kinetic energy for each panel are drawn at $\bar{u}_{m}^{2} / 3,2 \bar{u}_{m}^{2} / 3, \ldots, 2$.

approximately $4 D$, and the amount of energy decreases as the breakdown locations move downstream with increasing $U_{\text {red }}$.

\section{Wavy instability modes}

\subsection{Linear instability}

In figure 6 , it is observed that $R e_{c}$ for the period-doubling mode increases rapidly as $U_{\text {red }}$ decreases to values below two. Also, in figure 5, a second leading mode is observed at wavenumber $k=4$. It transpires that for a range of reduced velocities $U_{\text {red }}<2$, the set of modes $k>1$ that are stable at higher reduced velocities grow to dominate, as exemplified in figure 10 which shows the spectrum of Floquet multipliers at $U_{\text {red }}=0.875, R e=350$, where both $k=3$ and $k=4$ are unstable modes, and the one at $k=3$ dominates. Note that now the dominant multipliers are real and positive, and hence the modes are synchronous with the base flow. 


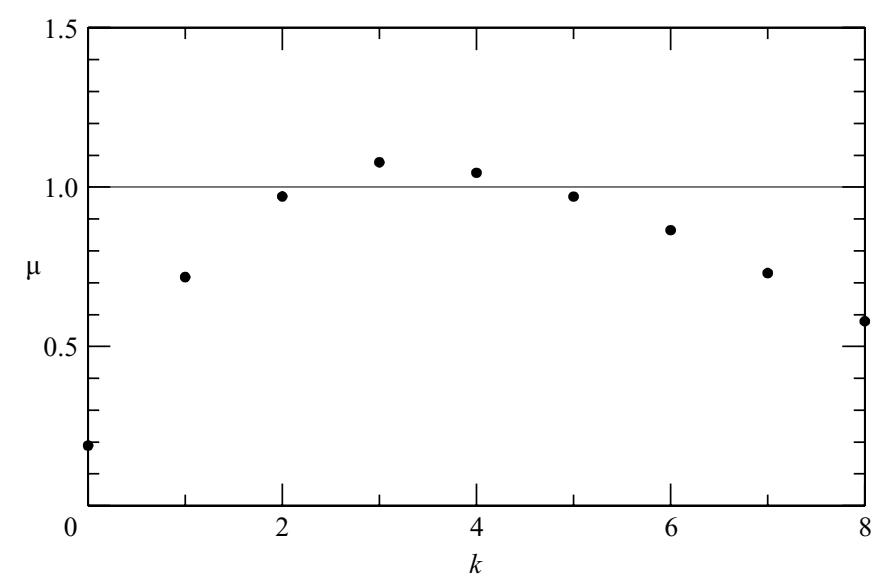

FIGURE 10. Azimuthal wavenumber spectrum of Floquet multipliers at $U_{\text {red }}=0.875$, $R e=350$. Wavy modes with both $k=3$ and $k=4$ are unstable.

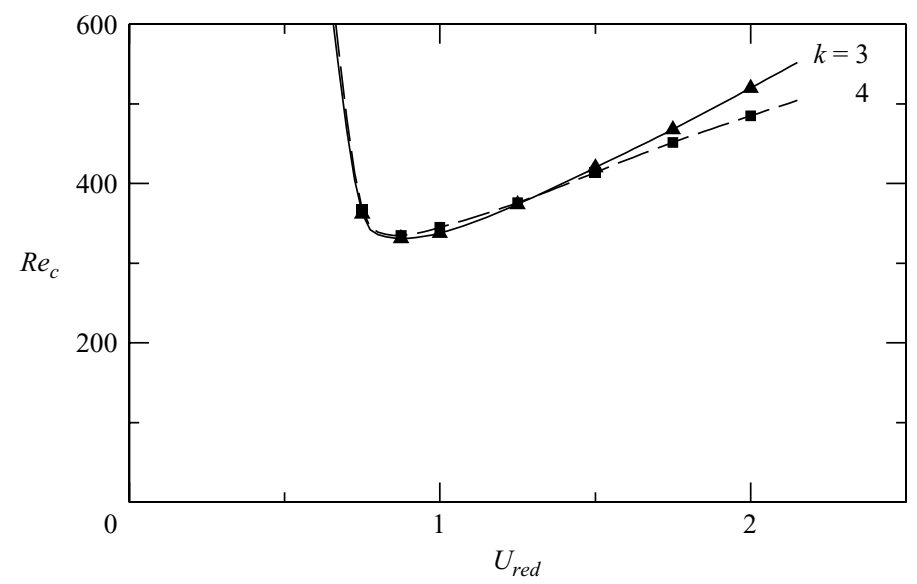

FIGURE 11. Critical curves for the wavenumber $k=3$ and $k=4$ wavy modes.

The marginal-Re stability curves for the $k=3$ and $k=4$ modes compete for dominance, as shown in figure 11 where it is seen that for $U_{\text {red }} \gtrsim 1.5$, the $k=4$ mode is the least stable, while at lower reduced velocities the $k=3$ mode becomes the slightly less stable of the pair. Like the period-doubling mode, the wavy modes have an 'optimal' reduced velocity (now near $U_{\text {red }}=0.875$ ); at lower values, the marginal stability Reynolds numbers increase rapidly with falling $U_{\text {red }}$, whereas in the high- $U_{\text {red }}$ limit, there is again an approximately linear variation of $R e_{c}$ with $U_{\text {red }}$ for both modes.

Figure 12 (see p. 69) uses isosurface visualization to illustrate the structure of these wavy modes at $U_{\text {red }}=0.875, R e=325$, slightly below the marginal stability value of $R e_{c}=335$ for the $k=3$ mode. We have used an isosurface of a positive value of the discriminant $\mathscr{D}$ of the velocity gradient tensor for the base flow to show the locations of vortex rings, while the linear instability modes are shown using equal-magnitude positive/negative values of streamwise vorticity component. Note that in this figure there is by definition no distortion of the base flow vortices, which have been shown to reveal the spatial relationship between the instability modes and the base flow. In 

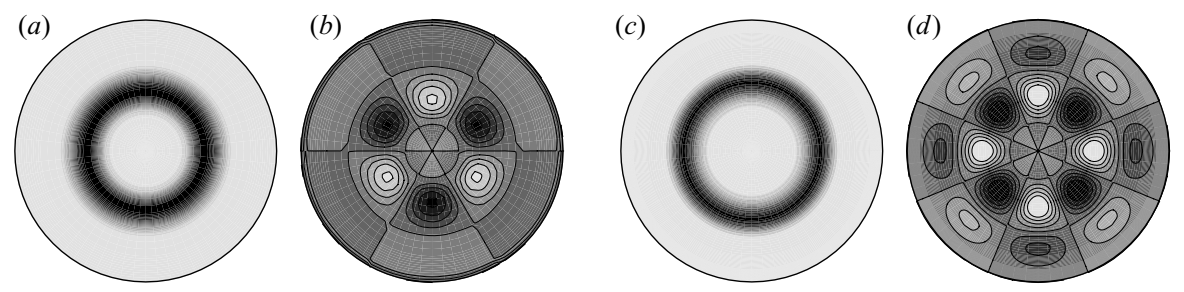

FiguRE 13. Structure of the wavy modes in cross-section. for $U_{\text {red }}=0.875, R e=325$, phase point $t=j T$. (a) Contours of velocity gradient tensor discriminant $\mathscr{D}$ (black, positive) for the base flow at $z / D=7.75$ and $(b)$ contours of the $k=3$ Floquet mode radial velocity component at the same axial station - nine contours drawn equispaced over an arbitrary but equal-magnitude, positive/negative range. $(c, d)$ Equivalent contours at axial station $z / D=4.95, k=4$ Floquet mode.

figure $12(a)$, the $k=3$ instability mode can be seen to begin growing in an isolated manner on the second vortex downstream of the stenosis. Therefore, this is a type of wavy vortex ring instability. It seems, however, that as the modal energy grows downstream there is significant axial linkage between the three-dimensional instability growing on sequentially created rings. Since the sign of streamwise vorticity in the instability is constant along an axial traverse at fixed radius, the axial linkage indicates cooperation between instabilities of successive rings. If anything, this phenomenon appears stronger for the $k=4$ instability seen in figure $12(b)$.

More detail of the cross-sectional structure of the base flow and instability modes can be seen in figure 13, again for $U_{r e d}=0.875, R e=325, t=j T$. Figures $13(a)$ and $13(b)$ show, respectively, a contour of velocity gradient discriminant $\mathscr{D}$ in the base flow and a contour of radial velocity component of the $k=3$ Floquet mode, at axial location $z / D=7.75$, while figures $13(c)$ and $13(d)$ show corresponding contours for the $k=4$ mode, at axial location $z / D=4.95$. While contours of $\mathscr{D}$ for the base flow indicate vortex structures apparently confined to $r / D \lesssim 0.3$, the spread of positive azimuthal vorticity on the same sections is rather more diffuse, reaching from $r / D=0$ to $r / D \approx 0.45$ with a maximum near $r / D=0.23$. The radial structure of each Floquet mode contains two nodes, which conforms to the predictions of Widnall et al. (1974) for short-wavelength instabilities on isolated unconstrained vortex rings, even though the current rings cannot be considered slender (of small core/radius ratio) as was the case in their analysis, and are also contained within a tube.

The analysis of Widnall et al. (1974) may be summarized by figure 4 of their paper, which shows the most unstable wavenumber as a function of dimensionless vortex ring speed $\tilde{V}=V_{0} /(\Gamma / 4 \pi R)$, where $V_{0}$ is the vortex propagation speed, $\Gamma$ is its circulation, $R$ is the ring radius. Their computations were carried out for a number of assumed core vorticity distributions; a smooth distribution had the closest match to their experimental results, which, however, always gave slightly higher values of instability wavenumber. To compute a representative $\tilde{V}$, we use $V_{0}=3 \bar{u}_{m}$ (see our figure 4), and for $U_{\text {red }}=1$ have computed $\Gamma=5.46 \bar{u}_{m} D$ for the first identifiable vortex ring, centred near $(z / D=3.3, r / D=0.29)$, in figure $3(b)$. This gives $\tilde{V}=3 /(5.46 / 4 \pi \times 0.29)=2.00$. Extrapolating the smooth-core data in figure 4, Widnall et al. (1974) to $\tilde{V}=2$, we find an instability wavenumber of approximately 3 ; extrapolating the experimental data gives approximately 4 . In conjunction with the fact that we obtain the same radial structure of instability modes as Widnall et al. (1974), this agreement with our Floquet-mode wavenumbers supports the proposition that our wavy modes can be considered to be a type of Widnall vortex-core instability. 

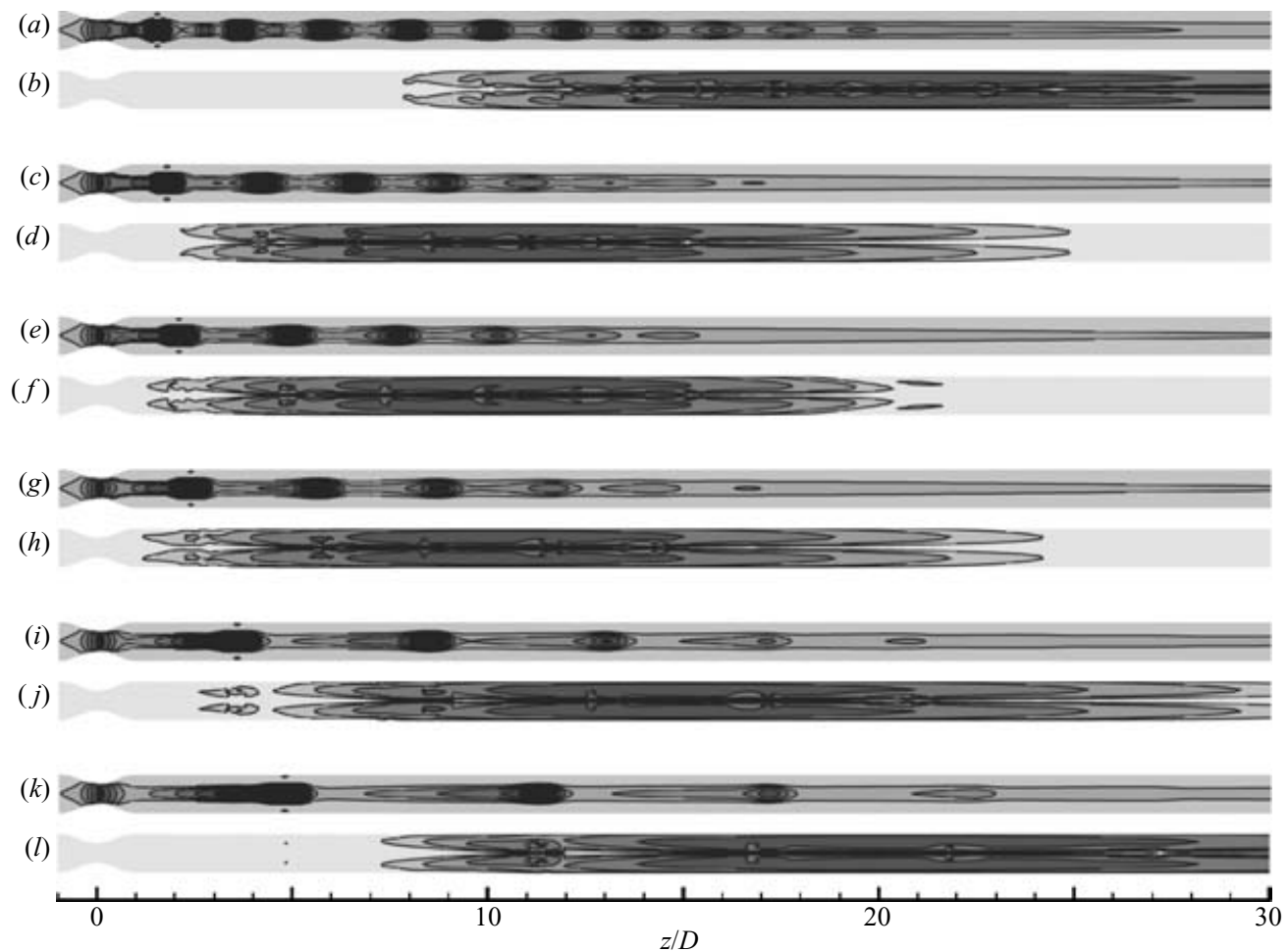

FIGURE 14. Contour plot pairs showing instantaneous energy in the base flow and logarithm of energy in the wavy-ring Floquet instability mode at an azimuthal wavenumber of $k=3$ for: $(a, b) U_{\text {red }}=0.65, R e=600 ;(c, d) U_{\text {red }}=0.75, R e=350 ;(e, f) U_{\text {red }}=0.875, R e=325$; $(g, h) U_{r e d}=1, R e=339 ;(i, j) U_{\text {red }}=1.5, R e=425 ;(k, l) U_{\text {red }}=2, R e=500$. Results are all close to marginal stability and shown at phase point $t=j T$. Contour levels as for figure 7 .

Figure 14 shows pairs of plots of contours of energy in the base flows and the logarithm of energy in the $k=3$ Floquet modes for a progressive increase in reduced velocity. The modal energy contours in each case show that for these modes, energy is always spread in an apparently connected manner across a number of base flow pulses/vortices, which, as noted above, is taken again to be indicative of a cooperative mechanism. The optimum $U_{\text {red }}$ (near $U_{\text {red }}=0.875$, figure $14 e, f$ ) is, as was the case for the period-doubling mode, again associated with modal energy occurring at a minimum distance from the stenosis.

\subsection{Nonlinear evolution}

Under nonlinear evolution from a base flow seeded with the instability mode, a sequence of events is found similar to the case for the period-doubling mode. Starting with initial exponential growth, there is an initial nonlinear saturation with the peak energy occurring in an axial location similar to that for the Floquet mode, and this is followed by a second slow saturation to the asymptotic state. Again the asymptotic state possesses a localized turbulent breakdown, and subsequently the slow second saturation is associated with an upstream migration to a limit of the site of this breakdown.

Figure 15 (see p. 69) illustrates the three-dimensional transitional flow at $U_{\text {red }}=1$, $R e=350$, promoted by an instability in the $k=3$ mode. In figure $15(a)$, at 


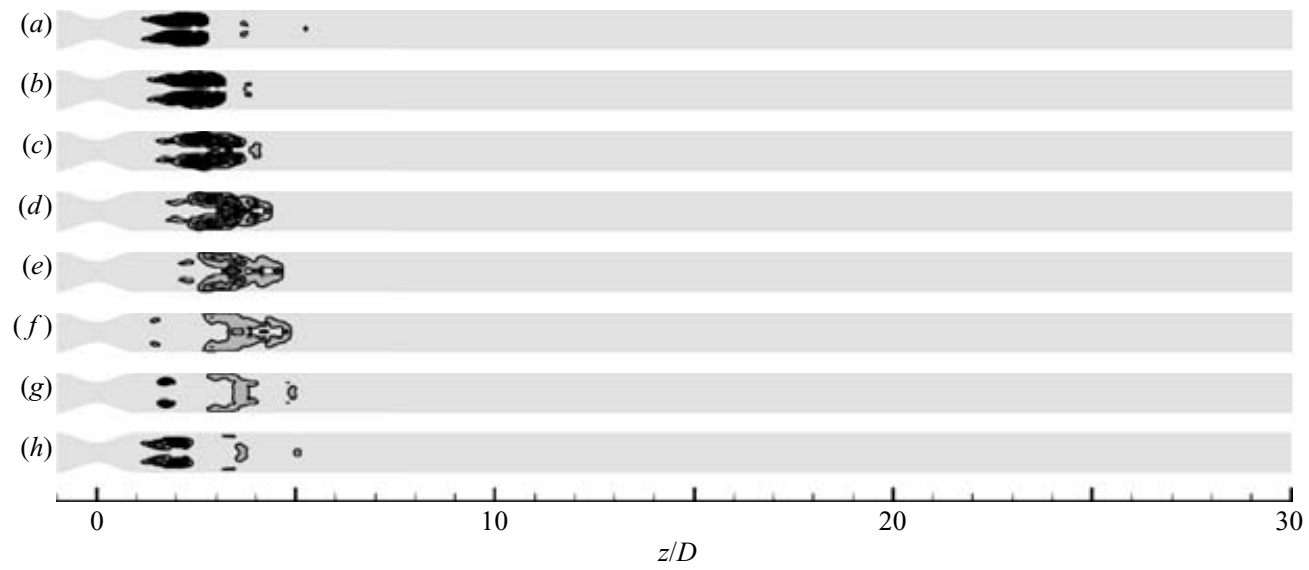

FIGURE 16. Contours of phase-averaged turbulent energy $q^{\prime}$ on a slice through the pipe centreline for the asymptotic state at $U_{r e d}=1, R e=350$ for phases: (a) $t=j T ;(b)$ $t=(j+1 / 8) T ;(c) t=(j+2 / 8) T ;(d) t=(j+3 / 8) T ;(e) t=(j+4 / 8) T ;(f) t=(j+5 / 8) T$; (g) $t=(j+6 / 8) T ;(h) t=(j+7 / 8) T$. Contour levels as for figure 9 .

the end of the first weakly nonlinear saturation, we see a perpective view looking upstream to a sequence of vortex rings, where the ring nearest the viewpoint is highly distorted, and has three-fold azimuthal symmetry. The similarity to previously published visualizations of comparatively low-wavenumber smoke-ring instabilities (see e.g. figure 5 of Widnall et al. 1974) is striking. Figure 15 (b) shows a different view at the same stage of nonlinear evolution, and we can see that further downstream, the ring generated immediately prior to that which is highly distorted but also highly symmetrical in figure 15(a) undergoes a complicated (and highly dissipative) breakdown. Figure 15(c) shows results from the same simulation, but many pulse cycles later, after the system has reached its asymptotic state. Here, three-dimensional instability reaches upstream to begin vortex ring distortion almost immediately following detachment, and this is linked directly to the breakdown of the previously created vortex ring.

Similar to the data processed for the period-doubling mode in figure 9, we have also considered the contours of phase-averaged turbulence energy (after the mean and periodic base flow has been removed) for the wavy mode. Figure 16 shows contours of phase-average (and also azimuthal-average) turbulent energy $q^{\prime}$ for the asymptotic state at $U_{\text {red }}=1, R e=350$. Contour levels are the same as for figure 9. Once again we observe how the energy is confined in a region very close to the stenosis, $z / D$ in the range $1-5$ as compared to the large spatial extent of the Floquet mode.

\section{Interaction with convective shear-layer instability}

As was noted in $\S 1$, a feature of previous experimental studies of stenotic flows (e.g. Khalifa \& Giddens 1981; Ojha et al. 1989) is the appearance of shear-layer instabilities. Because a well-resolved numerical study provides a much lower level of extraneous input (i.e. noise) than is possible in even the most well-controlled physical experiment, we do not normally observe shear-layer oscillations unless the flow has become turbulent, or forcing is explicitly introduced to drive them. We observed (Sherwin \& Blackburn 2005) spontaneous shear-layer oscillations in DNS of a turbulent stenotic flow at $R e=750$ with a steady Hagen-Poiseuille inflow, and 


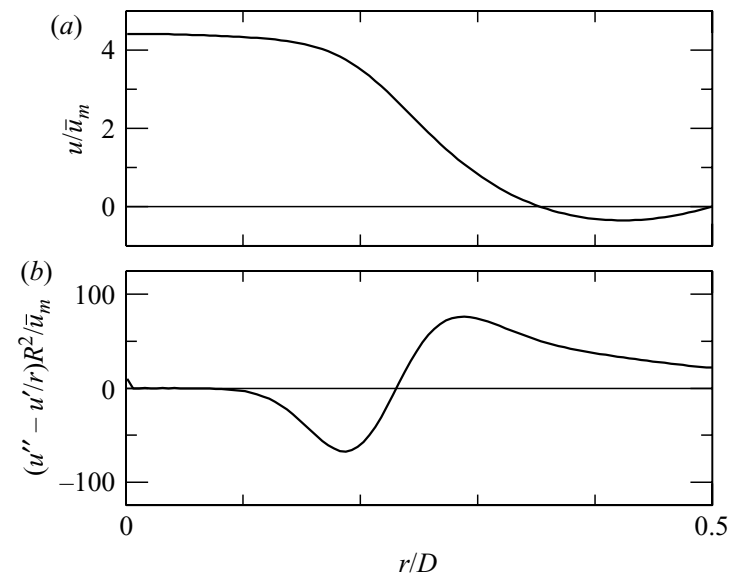

FIGURE 17. Local analysis of inviscid instability for the axial velocity profile of steady flow at $R e=700, z / D=2.5$. (a) Axial velocity profile; (b) profile for the cylindrical-coordinate equivalent of Fjørtoft's criterion (Kumaran 1996, Proposition 2).

made a more detailed study of the presence and effect of shear-layer oscillations driven by a high-frequency inflow perturbation on a two-harmonic pulsatile flow at $U_{\text {red }}=5$. For the latter flow, the effects of the shear-layer oscillations on the Floquet instability were minimal, which was thought to be because the Floquet mode's most energetic region was physically remote from the location of the shear-layer instability. In general, we may expect pulsatile flows of higher reduced velocity to be more susceptible to shear-layer oscillations, because, as outlined in $\S 3$, shear layers that appear in the wake of vortex rings become more extensive at large pulse periods. The frequencies at which the shear layers are excited are usually well above those of the underlying pulsatility, related to the fact that the shear layers are thin compared to the tube diameter.

In the present section, we return to the study of convective shear-layer instability of these flows energized through high-frequency inflow forcing. Our method will be relatively heuristic, being based largely on axisymmetric DNS, coupled with our Floquet analysis technique when required in specific cases. Since, by definition, a convective instability will eventually wash out of the domain in the absence of perturbation, we drive it continuously by adding a small-amplitude harmonic streamwise perturbation to the inflow boundary condition. It is found that over a comparatively narrow frequency/reduced velocity band, this type of perturbation excites oscillations in separated shear layers downstream of the stenosis throat and may induce shear-layer roll-up. We recognize that recent adjoint-based methods advanced for the study of non-normal global modes and optimal growth problems (see e.g. Chomaz 2005) would provide a more complete formal basis for this study.

\subsection{Convective instability of steady flow}

Axisymmetric steady flow in our stenotic geometry is susceptible to shear-layer instability. The flow may naively be expected to possess instability because radial profiles of axial velocity beyond the separation point immediately downstream of the stenosis, and until the Hagen-Poiseuille profile is restored, all show a point of inflection. Using the cylindrical-coordinate equivalent of the Fjørtoft criterion derived by Kumaran (1996, Proposition 2), we demonstrate in figure 17 that, under inviscid assumptions, these flows satisfy a necessary condition for instability, since at the wall 
(a)

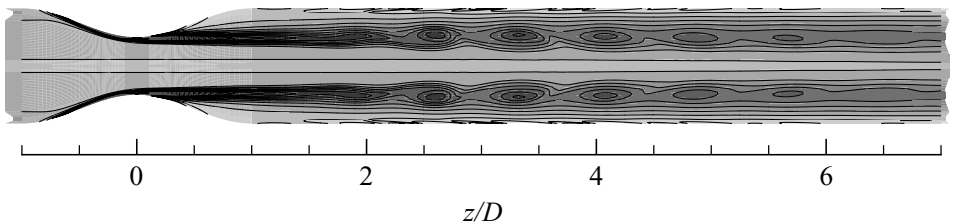

(b)

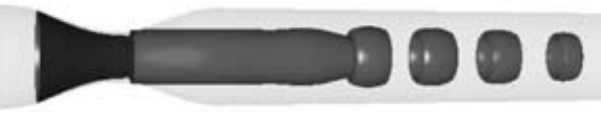

FigURE 18. (a) Contours and $(b)$ isosurface of instantaneous azimuthal vorticity $\left(\omega_{\theta}=25 \bar{u}_{m} / D\right)$ for the axisymmetric convective instability of steady flow at $R e=700, U_{\text {red }}=0.3$, near peak sensitivity.

$u^{\prime}<0$, and there is a region where $u^{\prime \prime}-u^{\prime} / r \geqslant 0$ (here, prime signifies differentiation with respect to the radial coordinate).

For the present viscous flow, we employ the simple expedient outlined above of adding a small-amplitude high-frequency harmonic to the inflow, so that it is described by

$$
\bar{u}(t)=\bar{u}_{m}[1+0.001 \sin (2 \pi t / T)] .
$$

The perturbation is at a level that is visibly imperceptible compared to the underlying flow; however, it excites a convective instability in the separated shear layer, whose pointwise amplitude may be much larger than the perturbation. The visual characteristics of the resulting flows are exemplified in figure 18, where the Reynolds number of the bulk flow is $R e=700$ (below the critical global instability Reynolds number for steady flow of 722 found by Sherwin \& Blackburn 2005) and the reduced velocity of the perturbation is $U_{\text {red }}=0.3$. Note that the shear layer rolls up into a set of discrete vortices. The mean vortex spacing at formation is $\Delta z / D \approx 0.7$, rising slowly downstream to approximately 0.8 before diffusion takes over and the vortices dissipate at $z / D \sim 6$.

In order to quantify this behaviour, we use as a measure of response the cyclemaximum kinetic energy in the perturbation flow,

$$
Q_{\max }^{\prime}=\max _{T} 0.5 \int_{\Omega} r\left(\boldsymbol{u}-\boldsymbol{u}_{\text {base }}\right)^{2} \mathrm{~d} \Omega,
$$

where $\Omega$ is the extent of the two-dimensional computational domain, normalized by the domain-integral of the kinetic energy in the corresponding steady (unperturbed, base) flow, $Q$. This normalized quantity is shown as functions both of perturbation reduced velocity and mean flow Reynolds number in figure 19. It can be seen that the shear layer is susceptible over a range of reduced velocities that broadens as Reynolds numbers increase, and also that the responses peak near $U_{\text {red }} \approx 0.4$.

\subsection{Convective instability of pulsatile flow}

Now we examine the effect of a high-frequency perturbation applied on top of base flows that are already pulsatile with $U_{p m}=1.75$, i.e. those that served as the bases for the work of $\S \S 3-5$. In order to contain the investigation to a manageable size, we have chosen three exemplar base flow reduced velocities: $U_{\text {red }}=0.875$, the most susceptible for wavy instability; $U_{\text {red }}=3.25$, the most susceptible for period-doubling instability; and $U_{\text {red }}=10$, the highest included in the present work. The corresponding Reynolds numbers were set, respectively, at 350, 400 and 600, in each case slightly greater than 


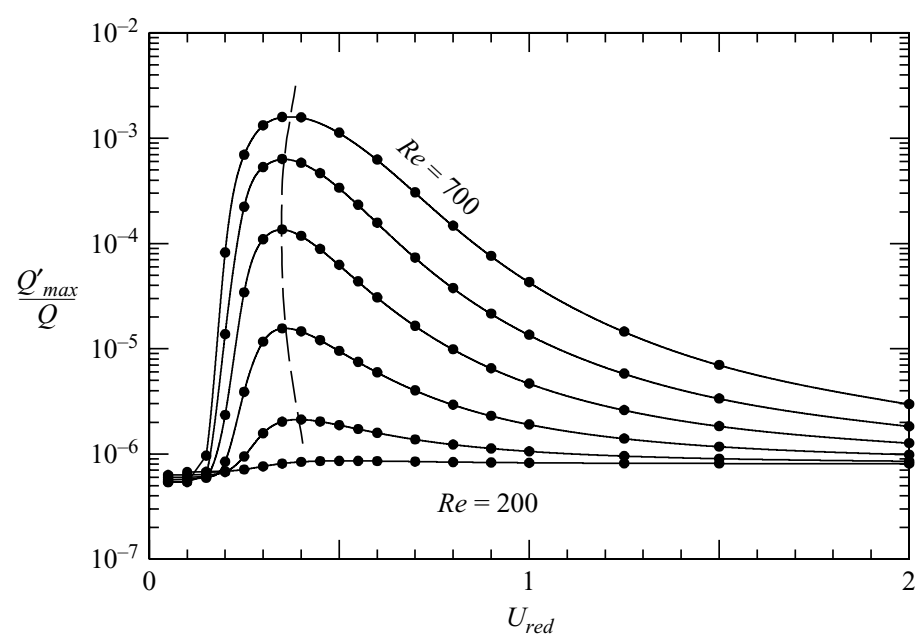

FIGURE 19. Convective instability results for axisymmetric steady flow. These show the domain integral of kinetic energy in the difference between the perturbed flow and the steady flow, normalized by the domain integral of energy in the corresponding steady flow, as functions of reduced velocity and Reynolds number (in steps of 100, from 200 to 700 ).

the marginal-Floquet-stability value. We recall that according to the results presented in $\S 3$, all the unperturbed flows will produce a single vortex ring per pulse period, but that as reduced velocities increase, we can expect that there will be an increasingly long shear layer left in the wake of this 'vortex front'. The bulk inlet flow is now described by

$$
\bar{u}(t)=\bar{u}_{m}[1+0.75 \sin (2 \pi t / T)+0.001 \sin (2 \pi n t / T)] .
$$

To help reduce the parameter space and for simplicity, the high-frequency perturbation is chosen to be temporal harmonic $n$ of the base flow. Further, we have considered only the fixed phase relationship described above. If the reduced velocity of the underlying pulsatile base flow is described by $U_{\text {red }}$ (base) $=\bar{u}_{m} T / D$, the reduced velocity of the perturbation can also be written as $U_{\text {red }}(\varepsilon)=U_{\text {red }}$ (base) $/ n=\bar{u}_{m} T / n D$.

\subsubsection{Effect on axisymmetric flow}

Initially, the response to the perturbation forcing was examined as an axisymmetric problem. The effect of shear-layer excitation is quantified in a similar fashion as employed in (6.2) for steady base flows, albeit generalized so that at any point in the pulse period $T$, the perturbation flow is computed with respect to the relevant instantaneous unperturbed single-harmonic flow. Likewise, the normalizing energy, $\hat{Q}$, is now the peak domain-integral kinetic energy in the unperturbed flow. The response amplitudes are shown in figure 20. Clearly, the sensitivity to perturbation grows with reduced velocity of the base flow and as for the steady flow, there exists a perturbation reduced velocity at which each flow is most sensitive. For the pulsatile flows, this is of order 0.2, approximately half the value for steady flow.

Since in this study when increasing the base flow reduced velocities we have also increased Reynolds number, we cannot state conclusively that the increased sensitivity stems solely from increase in reduced velocities. However, observation of the perturbed flows shows that the response is more significant in the extended shear layers that exist in the wake of a vortex ring at higher base flow reduced velocities. Thus, following 


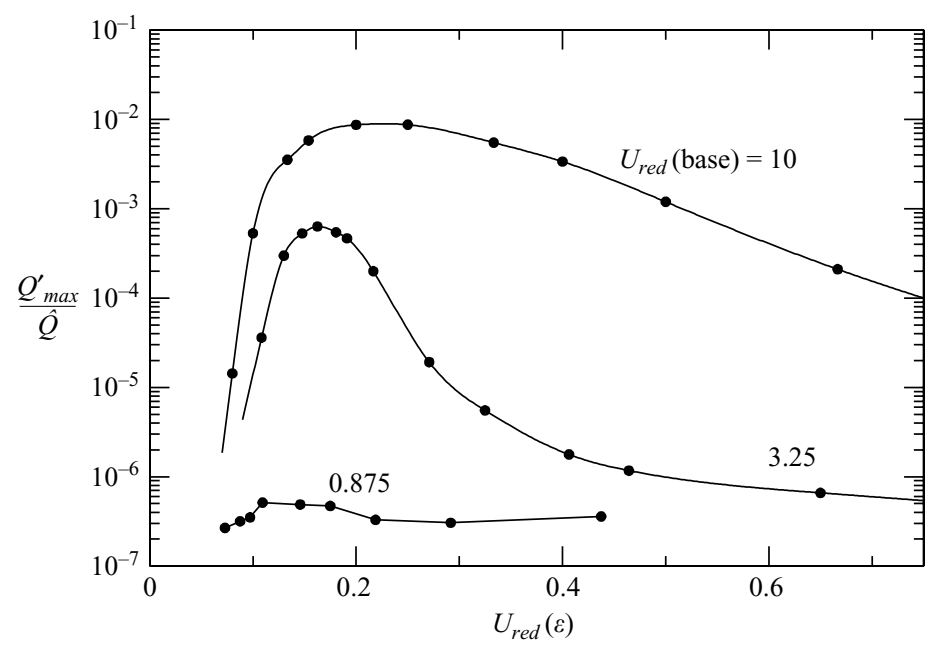

FIGURE 20. Convective instability results for axisymmetric pulsatile flows. Peak domainintegral energy in the perturbation flow $Q^{\prime}$ (normalized by peak energy in the unperturbed flow, $\hat{Q}$ ) when a high-frequency low-level harmonic forcing at reduced velocity $U_{\text {red }}(\varepsilon)$ is added to the inlet of three pulsatile base flows. Reynolds numbers for the three base flows at $U_{\text {red }}=0.875,3.25$ and 10 were, respectively, 350, 400 and 600 .

the reasoning outlined at the start of $\S 6.2$, we may expect that at a given Reynolds number, pulsatile flows of higher reduced velocity should be more responsive to convective instability. As also noted above, the range of excited perturbation reduced velocities also broadens with an increase in reduced velocity of the base flow.

\subsubsection{Interaction with Floquet instability}

We note that in the three cases considered, the perturbation reduced velocity for peak sensitivity to convective instability, $U_{\text {red }}(\varepsilon) \approx 0.2$, lies well below (i.e. at a shorter period than) the reduced velocities for either the period-doubling or wavymode Floquet instabilities. However, it is interesting to examine the effect of the high-frequency perturbation on Floquet instability, where the base flow for stability analysis is now the high-frequency-perturbed axisymmetric pulsatile flow. Analysis has been carried out for the three cases with a perturbation reduced velocity corresponding approximately to the peak values found in figure 20: for $\left(U_{\text {red }}(\right.$ base $)=0.875, R e=$ $350), n=7$, i.e. $U_{\text {red }}(\varepsilon)=0.125$, wavenumber $k=4$; for $\left(U_{\text {red }}(\right.$ base $\left.)=3.25, \operatorname{Re}=400\right)$, $n=20$, i.e. $U_{\text {red }}(\varepsilon)=0.1625, k=1$; and for $\left(U_{\text {red }}\right.$ (base) $\left.=10, R e=600\right), n=50$, i.e. $U_{\text {red }}(\varepsilon)=0.2, k=1$. While for $U_{\text {red }}$ (base) $=0.875$ and 3.25 there is almost no effect on Floquet multipliers, which are the same to three significant figures, with or without the perturbation, there is a large destabilizing effect for $U_{\text {red }}=10$, since the multiplier increases by approximately an order of magnitude from $\mu=-1.18$ to $\mu=-12.7$ when the perturbation is present. (By mesh $N_{p}$-refinement, we have checked that these values are resolution-independent.) Note that the period-doubling character of this instability is robust to the applied perturbation, since the multiplier remains negative. In figure 21, we show base kinetic energies of both base flows and Floquet modes for both the unperturbed case $(a-d)$ and perturbed case $(e-h)$, for phases $t=j T$ and $t=(j+1 / 2) T$.

A number of features can be seen in figure 21. The first is that (especially for the unperturbed flow) the Floquet mode is most energetic near the leading edges of 


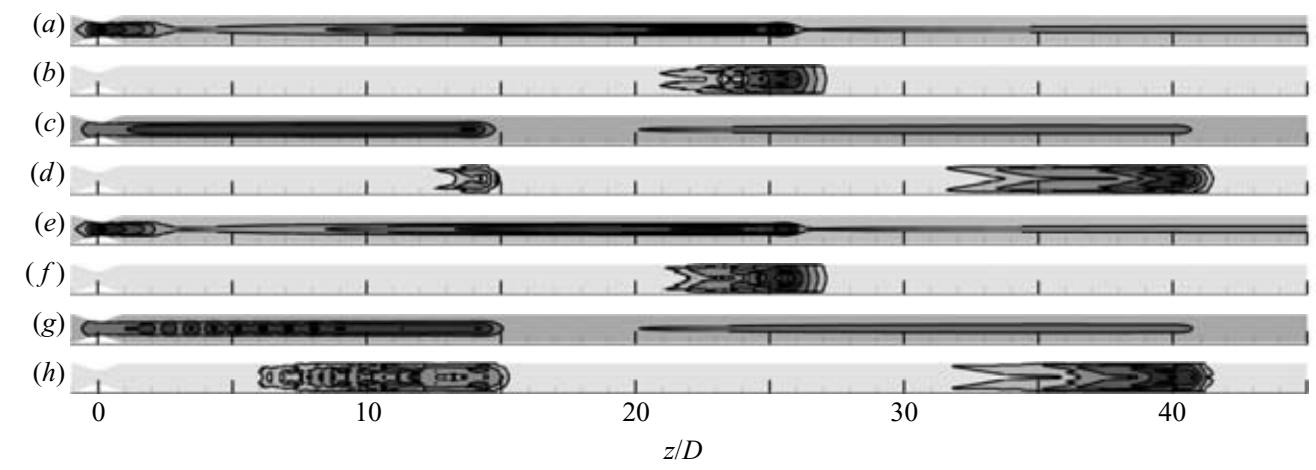

FIGURE 21. The effect of high-frequency low-amplitude perturbation on Floquet instability for $U_{\text {red }}=10, R e=600$. Contour plot pairs showing instantaneous energy $q$ in the base flow (top of pair) and logarithm of energy in the period-doubling Floquet instability mode (bottom of pair) on a plane containing the tube centreline. $(a-d)$ The case without perturbation, where $\mu=-1.18$, showing the base flow and Floquet instability mode for $(a, b), t=j T ;(c, d)$, $t=(j+1 / 2) T$. For $(e-h)$, high-frequency perturbation is added to the inlet flow, changing the Floquet multiplier to $\mu=-12.7$. Observe that the perturbation has most effect on both the base flow and mode shape for $(g, h)$, when $t=(j+1 / 2) T$. Base flow contour levels are drawn at 2, 4, 6, 8 and $10 \bar{u}_{m}^{2}$ in $(a)$ and $(e)$ and at 3,12 and $20 \bar{u}_{m}^{2}$ in $(c)$ and $(g)$. Eigenmode normalization and contour levels as for figures 7 and 14 .

pulse fronts (i.e. near the leading vortex rings), for both $t=j T$ and $t=(j+1 / 2) T$. The second is that the base flow is more energetic at phase $t=(j+1 / 2) T$ than at $t=j T$ (this is not immediately visually apparent, but follows from consideration of the different contour levels applied to the base flows at phases $t=j T$ and $t=(j+1 / 2) T)$. Thirdly, in common with the Floquet analysis results of figures 7 and 14, modal energy grows as the disturbance moves downstream, but this occurs as the corresponding base flow energy dissipates; eventually the modal energy also falls, but here that must occur outside the computational domain. Perhaps the most important feature is that while the high-frequency perturbation is always present, its effect is only obvious here - in both the base flow and the Floquet mode - for phase $t=(j+1 / 2) T$, when the extended shear layer is more energetic, in the wake of the vortex front closest to the stenosis. Clearly, the effect of the perturbation on the base flow is greatest within this extended shear layer, which at $t=(j+1 / 2) T$ (figure $21 \mathrm{~g}$ ) has rolled up into a set of concentrated vortices in much the same fashion as seen in figure 18 for the perturbed steady inflow. A significant increase in the axial extent of the Floquet mode energy in this vicinity can be seen in figure $21(h)$.

\subsubsection{Nonlinear response via DNS}

To conclude this section, the effect of applying the high-frequency perturbation on the inflow for DNS is investigated for $\left(U_{r e d}=10, R e=600\right)$. Restarting from the asymptotic state for the unperturbed flow obtained for these control parameters in $\S 4.2$, the high-frequency perturbation was added to the inflow, the simulation run for another 10 pulse cycles, followed by phase-averaging for 15 cycles. Contours of phase-averaged turbulent energy for this flow are shown in figure 22, which may be compared to those of figure $9(d)$. It is immediately apparent that the effect of the perturbation has been to translate the location of turbulent breakdown yet further upstream towards the stenosis, approximately halving the distance between the stenosis and the breakdown from order $10 D$ to order $5 D$. The effect of shear-layer oscillations in the phase-averaged turbulent energy is most evident for $t=(j+1 / 4) T$. 


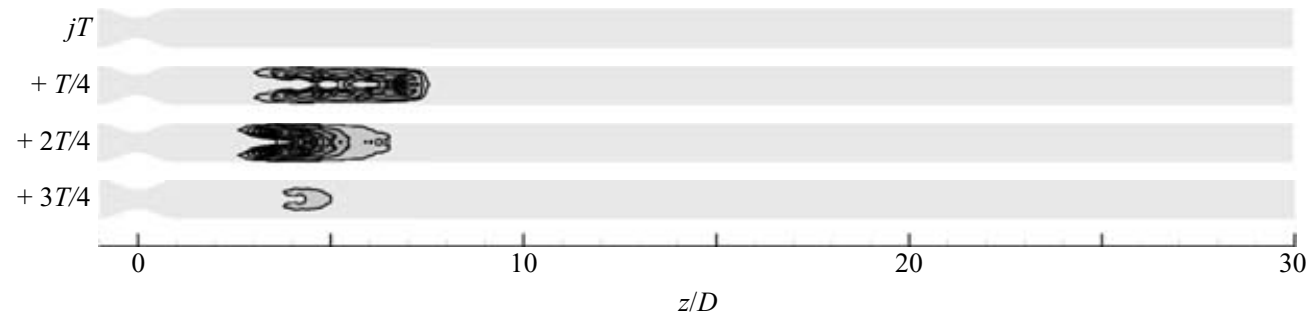

FiguRE 22. Contours of phase-averaged turbulent energy on a slice through the pipe centreline at four temporal phases for the asymptotic state in DNS at $U_{\text {red }}=10, R e=600$, with added high-frequency low-amplitude perturbation. Contour levels as for figure 9 ; cf. figure $9(d)$.

Response to the convective instability for $\left(U_{\text {red }}=10, R e=600\right)$ is large enough that it becomes almost impossible to discern the underlying period-doubling nature of the Floquet instability in the DNS results. This is not true in the absence of perturbation, where period-doubling is evident either visually (as for figure 8), or by detailed examination of velocity time series extracted at locations within the turbulent breakdown. When the perturbation is present, comparatively large high-frequency velocity fluctuations (albeit with significant bandwidth) associated with shear-layer oscillations can mask the effect. Naturally, the magnitude of the convective instability response and the importance of this masking effect will vary with the amplitude at which the convective instability is driven.

\section{Discussion}

Just as the most significant visual features of the stenotic pulsatile base flows in this study are found to be vortex rings and extended separated shear layers, so also are the instabilities found to be those of vortex rings and separated shear layers. It is worth remarking that these can be regarded as walled-bounded flow counterparts of previously recorded unbounded flow instabilities. The period-doubling ring-tilting mode seems closely related to the phenomenon of bifurcation of pulsed jet flows (see e.g. Reynolds et al. 2003), while the wavy-core mode appears closely related to the short-wavelength instability of isolated vortex rings (see e.g. Widnall et al. 1974). The local convective instability of (steady jet) shear layers has received extensive analytical treatment (see e.g. Monkewitz \& Huerre 1982; Huerre \& Rossi 1998).

\subsection{Pulsatile flows}

For the simple single-harmonic base flows, each flow pulse creates a single vortex ring. Over the range of reduced velocity considered, these rings travel at approximately the same initial speed, $3 \bar{u}_{m}$, independent of pulse period, as shown in figure 4. Since the speed of an isolated vortex ring is proportional to its circulation (Saffman 1992, chap. 10), this carries the likely implication that the circulation of the vortex rings is also approximately independent of pulse period, even though the pulse-integral vorticity flux must increase with pulse period and hence reduced velocity. At larger reduced velocities, an extended separated shear layer trails in the wake of each ring; this shear layer should contain the remaining vorticity not rolled into the vortex ring, and so becomes longer as reduced velocities increase. We have noted the apparent similarity of this behaviour to that which is observed in the formation of single vortex rings (Gharib et al. 1998), where there is a universal 'formation number' that describes the maximum amount of circulation that will be carried by a vortex ring produced 

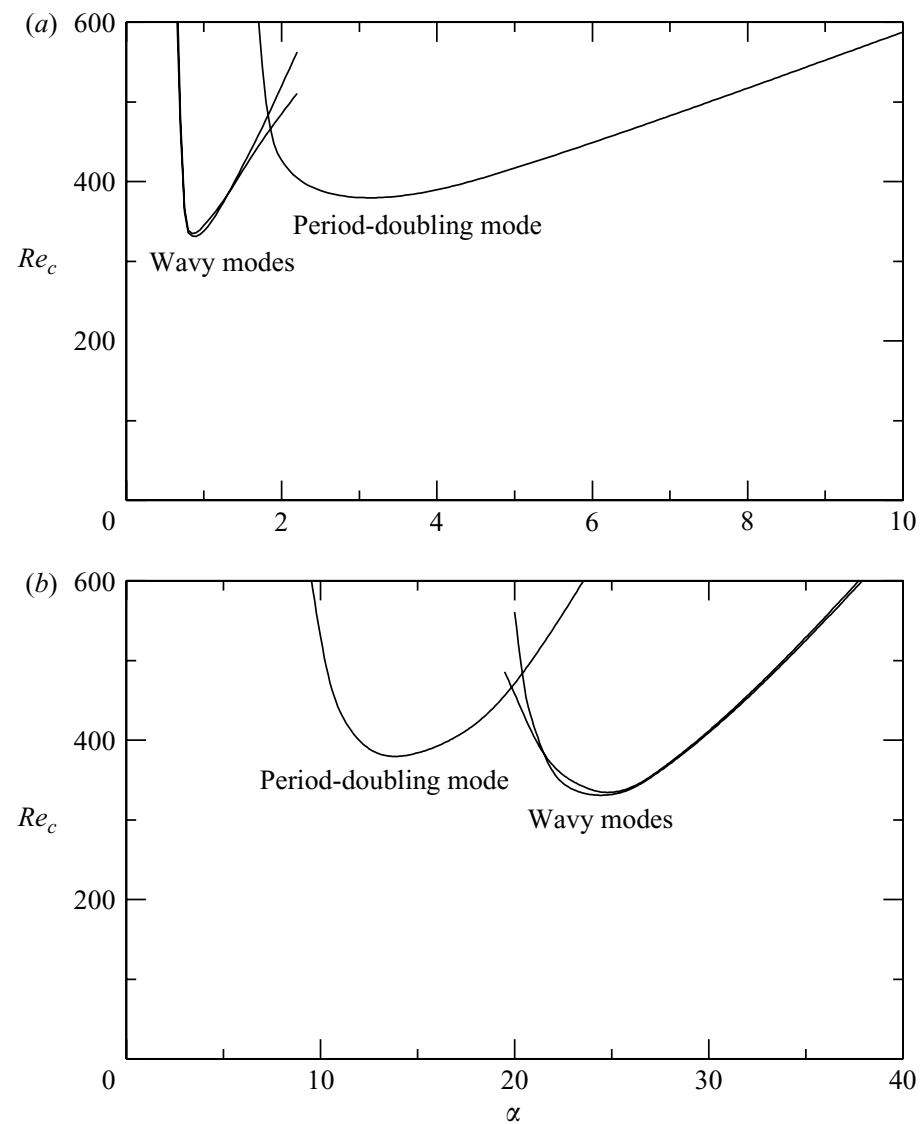

FIGURE 23. Summary curves of marginal Reynolds numbers for Floquet instabilities plotted as functions of $(a)$ reduced velocity and $(b)$ Womersley number.

by a translating piston, regardless of stroke above some minimum value; any excess vorticity trails behind the ring in an extended shear layer. We would expect that at sufficiently low pulse periods, vortex ring circulation and initial speed will fall, but see no evidence of this for reduced velocities as low as $U_{r e d}=\bar{u}_{m} T / D=0.5$.

The overall flow behaviour for complicated base flow pulsatility with many temporal harmonics is not yet known; however, it is likely that the basic features will be dominated by consideration of vortex rings and extended shear layers. In our earlier work, we considered a base flow with two harmonics (Sherwin \& Blackburn 2005, $\S \S 6.1 .2,6.4 .3)$. In that case, two vortex rings were formed per pulse period, with the stronger of the two overtaking and coalescing with the weaker ring some distance downstream of the stenosis, and, in the process, trailing an extended thin shear layer.

\subsection{Floquet instability and transition}

The two primary types of global instability modes that we have found are both associated with the vortex rings generated during each pulsatile cycle and also represent the largest length flow scales in the problem. Figure 23(a) shows a compilation of the marginal-Re stability curves for the wavy and period-doubling Floquet instability modes, plotted as functions of reduced velocity. At low reduced 
velocities, short wavelength vortex core instabilities dominate, and the connection to the analysis and experiments of Widnall et al. (1974) for similar instabilities of isolated and unbounded vortex rings was demonstrated in $\S 5.1$. The associated length scales are those of the vortex ring diameter and core size. At larger reduced velocities, an alternating, or period-doubling vortex-tilting type instability dominates. In this case, the more relevant length scales are likely to be the ring diameter and axial separation of successive rings. Similar instabilities, including both bifurcating and blooming pulsatile jets, have been recorded, but not as far as we know analysed, for unbounded flows. By definition, period-doubling cannot arise for an isolated vortex ring, but tilting, producing a divergence of path, is certainly possible.

The axial locations of peak Floquet mode energy vary with reduced velocity, but a common feature underlying all these instabilities appears to be a competition between an instability that grows with distance downstream of the stenosis (i.e. with time), while the energy in the vortex rings that feed the instability dissipates as they move downstream. Both the wavy and the ring-tilting instability modes have an 'optimal' reduced velocity at which the critical Reynolds number reaches a minimum; this value is $U_{\text {red }} \approx 0.875$ for both the $k=3$ and $k=4$ wavy modes, and $U_{\text {red }} \approx 3.25$ for the $k=1$ tilting mode. For both types of instability, the optimum coincides with the location of peak Floquet mode energy reaching its most upstream point (see figures 7 and 14).

We suggest that the existence of a minimum critical Reynolds number at an 'optimal' reduced velocity is, for both the vortex-tilting and wavy modes, the outcome of a competition between two opposing effects. The first effect stems from the fact that, at a fixed Reynolds number, vortex rings of the base flow survive for a greater time at higher reduced velocities (see figure 4). Since the Floquet instabilities derive their energy from these vortex rings, we may reasonably expect the Reynolds number required for marginal stability to fall with the increased vortex-ring lifetimes that occur as the reduced velocity of the base flow rises. The second effect comes about because the spacing between successive rings increases approximately linearly with reduced velocity. This increased spacing reduces the strength of cooperative coupling which we have noted for both kinds of Floquet instability. As a result, we may expect that the Reynolds number required for marginal stability would rise as reduced velocity increases. A balance between these two effects determines the location of the optimal reduced velocity. We note that for both the wavy and tilting modes, there is an approximately linear relationship between $U_{\text {red }}$ and $R e_{c}$ in the high- $U_{\text {red }}$ limits.

As pointed out in $\S 1$, the experimental observations and our previous numerical study are broadly consistent in predicting that when Reynolds numbers are high enough for transition to occur, this is manifested as pulse-locked turbulent bursts that are located some diameters downstream of the stenosis, and the flow then will apparently relaminarize further downstream. The present results support and extend those findings by showing a pulse-period dependence of the axial location of these turbulent bursts, brought about by self-destruction of vortex rings through either period-doubling or wavy instabilities. It is evident from an examination of figures 9 and 16 that the location of turbulent breakdowns in the asymptotic states moves downstream as reduced velocity, or equivalently pulse period, is increased. In fact, we might expect there to be a simple linear relationship between this location and the reduced velocity. In figure 24, we plot the axial location of the centroid of the distribution of $q^{\prime}$ (taken when the total turbulent kinetic energy is a maximum at one of the eight phase points) against reduced velocity, and it is seen that, indeed, such an approximately linear relationship exists. 


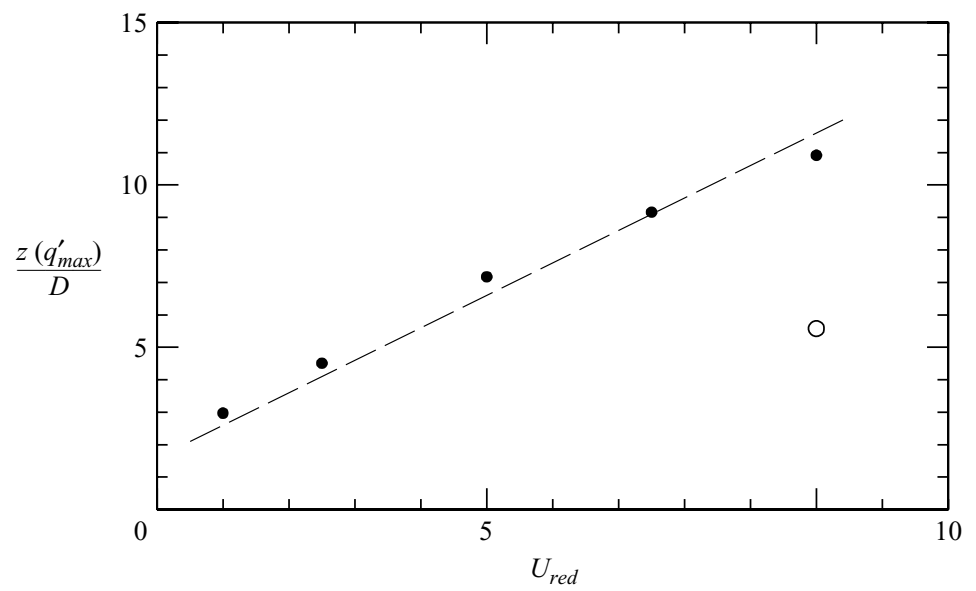

FIGURE 24. Axial location of the centroid of maximum turbulent energy (conditioned by phase averaging) in asymptotic states in the absence of high-frequency inflow perturbation $(\bullet)$. The dashed line has a slope of unity. At $U_{\text {red }}=10$, the single isolated datum (O) shows the result when high-frequency perturbation is added to the inflow.

\subsection{Convective shear-layer instability}

Extended shear layers that may be present in these flows at large pulse periods are susceptible to convective instabilities which can grow and die within a single pulse, even though driven continuously. Pulsatile flows of higher reduced velocity are more receptive to this type of instability simply because the shear layers become longer as pulse periods increase, as discussed in $\S 7.1$. The shear layer of steady stenotic flow is susceptible to convective instability at Reynolds numbers below the first critical global instability Reynolds number. We note again that the quantitative results obtained will be dependent on the nature and amplitude of the forcing used to drive the convective instability. In our essentially nonlinear (DNS-based) studies, the amplitude of high-frequency harmonic inflow forcing was maintained at $0.001 \bar{u}_{m}$.

There is little evidence that the convective instability will interact with the Floquet instability at the optimal reduced velocities for the onset of the instability (i.e. 0.875 and 3.25), principally because the trailing shear layers are less pronounced at these low values. However, there was a substantial destabilizing effect found at $U_{\text {red }}=10$, with the Floquet multiplier at $R e=600$ increasing in size from $\mu=-1.18$ to -12.7 when the convective instability received low-level high-frequency driving. Even though the Floquet instability for $U_{\text {red }}=10$ was excited by the convective instability, it still maintained its essential period-doubling nature.

When high-frequency perturbative forcing was added to the inflow of DNS of transitional flow at $\left(U_{\text {red }}=10, R e=600\right)$, the effect was to reduce the axial distance downstream from the stenosis at which turbulent breakdown occurs as compared to the unperturbed case. A demonstration of this change is shown in figure 24, where at $U_{\text {red }}=10$ the axial downstream length to turbulent breakdown has been approximately halved by the presence of the high-frequency perturbation.

In any physical experiment or application, inevitably some uncontrolled noise or high-frequency component will be present, and for pulsatile flows with extended shear layers, this may have a substantial influence on the observed results. However, we suggest that, at least for low-amplitude noise, the fundamental natures of large-scale instabilities, which are associated primarily with distortions and instabilities of vortex rings, are likely to survive. 


\subsection{Physiological application and relevance}

Figure $23(b)$ re-presents the same data as figure $23(a)$, but with the Womersley number as the independent variable, rather than reduced velocity. In the larger arterial vessels of the human cardiovascular system, physiologically reasonable parameters can be argued to be in the range $100 \leqslant R e \leqslant 600$ and $4 \leqslant \alpha \leqslant 25$ in rest conditions (see e.g. McDonald 1974; Nichols \& O'Rourke 1998). Since for a given pulse rate and blood viscosity, $\alpha$ increases directly with vessel diameter, large Womersley numbers are associated with the larger vessels. Although conditions may change with pathology or with exertion, an examination of figure $23(b)$ suggests that the instability modes examined here are directly relevant to human arterial flows where significant stenosis is present.

The flow rate waveforms observed in the human body cannot typically be represented by a single Fourier harmonic, but may instead contain between eight and sixteen significantly energetic harmonics. One or more of these may well be able to excite either or both of the two Floquet instability modes. In $\S \S 6.2 .2$ and 6.2.3, we have also highlighted that a high-frequency low-amplitude inflow perturbation to a base flow of relatively high reduced velocity can significantly influence the response of the primary instability, although the fundamental nature of the instability is typically maintained. In the case we have studied at $U_{\text {red }}=10$, the most receptive perturbation frequency was a fiftieth harmonic which is higher than the typical frequency content of a physiological waveform. However, at increased reduced velocity and Reynolds number, there was a significant broadening of the frequecy band that can lead to shear-layer oscillations (see figure 19). Therefore, even a perturbation at the sixteenth harmonic where the perturbation reduced velocity would be $U_{\text {red }}(\epsilon)=10 / 16=0.625$ is likely to promote a similar interaction. Our present results for the susceptibility to shear-layer instability, together with our earlier study of shear-layer instability in a two-harmonic pulsatile flow (Sherwin \& Blackburn 2005, §6.4.3), and in vitro flow visualizations (Cassanova \& Giddens 1978; Khalifa \& Giddens 1981; Ahmed \& Giddens 1983; Ojha et al. 1989), suggest that shear-layer oscillations may be common. Since shear-layer instabilities are convective in nature and hence dependent on the magnitude and frequency content of any imposed disturbance (owing, for example, to vessel wall movement, upstream flow separation, respiratory or external body motion) it is very difficult to comment further on their physiological importance relative to the vortex-ring instability mechanisms without significant additional information.

It is not uncommon for atherosclerotic stenoses to be found not as isolated instances, but serially along an arterial branch (Goldstein et al. 2000; Dodds \& Phillips 2003). We may speculate that this pathology is somehow related to our observations of localized turbulent bursts downstream of a stenotic contraction, with associated high temporal and spatial gradients of shear stress on endothelial cells, while the magnitude of stress would be lower than that existing in the stenosis throat itself. If such a linkage exists, one might expect that multiple inline stenoses are created serially in time, too, with each successive stenosis helping to create fluid-mechanical conditions conducive to growth of a downstream plaque.

\section{Conclusions}

Through a systematic parametric variation of a dimensionless pulse period for a simple pulsatile stenotic flow, we have shown that there are at least two kinds of global instability mode, both associated with vortex rings produced by flow pulsatility: a period-doubling mode that dominates at high reduced velocity, and a set of wavy modes that dominate at low reduced velocity. In addition, for steady flow, and a 
specific pulsatile flow considered, the separated shear layer is susceptible to convective instability at still lower reduced velocities. When the base flow reduced velocity is large enough to allow an extended shear-layer region behind the primary vortex ring, we have observed that high-frequency low-amplitude perturbations greatly enhance the rate of growth of the primary Floquet instability. This interaction of the convective instability with the Floquet mode may be relevant to physiological flows which may possess significant energy up to the sixteenth harmonic of the fundamental pulse frequency.

If Reynolds numbers are sufficiently high, there will be a region of localized turbulent breakdown downstream of a stenotic contraction, followed by relaminarization. Even though the location of greatest Floquet mode energy can be far downstream of the stenosis, the turbulent breakdown always eventually settles much further upstream, where more energy can be extracted from the pulsatile flow. Further, when introducing high-frequency perturbations to a case with higher base flow reduced velocity, the turbulent breakdown occurs significantly closer to the stenotic region as compared to the unperturbed case. In our original problem definition, we mentioned that adoption of a $75 \%$ stenosis is motivated by the observation that, in clinical practice, a blockage can be readily identified through medical imaging as it corresponds to a $50 \%$ reduction in arterial diameter. The current investigation has further determined that for this degree of stenosis, transitional flow leading to a turbulent breakdown is possible at physiologically relevant values of Reynolds number and non-dimensional pulse period.

In real physiological flows, geometric imperfections such as stenosis asymmetry and vessel curvature, along with vessel distensibility, will also play a role in determining the nature of transition. The present work does not examine these aspects, but we hope that it may help to provide a frame of reference for such studies.

This work was supported through the Merit Allocation Scheme of the Australian Partnership for Advanced Computing, and all three-dimensional DNS studies were computed at APAC's National Facility in Canberra, to whose staff we extend our thanks. Both authors gratefully acknowledge UK EPSRC grants GR/T02553/01 and EP/E006493 in financial support of visits made to the UK by H. M. B. S. J. S. would like to acknowledge financial support from the Royal Academy of Engineering in the form of a Global Research Award.

\section{REFERENCES}

Ahmed, S. A. \& Giddens, D. P. 1983 Velocity measurements in steady flow through axisymmetric stenoses at moderate Reynolds numbers. J. Biomech. 16, 505-516.

Ahmed, S. A. \& Giddens, D. P. 1984 Pulsatile poststenotic flow studies with laser Doppler anemometry. J. Biomech. 17, 695-705.

Akhavan, R., Kamm, R. D. \& Shapiro, A. H. 1991 An investigation of transition to turbulence in bounded oscillatory Stokes flows. Part 1. Experiments. J. Fluid Mech. 225, 395-422.

BARKLEY, D. \& Henderson, R. D. 1996 Three-dimensional Floquet stability analysis of the wake of a circular cylinder. J. Fluid Mech. 322, 215-241.

Berger, S. A. \& Jou, L.-D. 2000 Flows in stenotic vessels. Annu. Rev. Fluid Mech. 32, 347-384.

Blackburn, H. M. 2002 Three-dimensional instability and state selection in an oscillatory axisymmetric swirling flow. Phys. Fluids 14, 3983-3996.

Blackburn, H. M. \& Lopez, J. M. 2003 The onset of three-dimensional standing and modulated travelling waves in a periodically driven cavity flow. J. Fluid Mech. 497, 289-317.

Blackburn, H. M. \& Sherwin, S. J. 2004 Formulation of a Galerkin spectral element-Fourier method for three-dimensional incompressible flows in cylindrical geometries. J. Comput. Phys. 197, 759-778. 
Blackburn, H. M., Mansour, N. N. \& Cantwell, B. J. 1996 Topology of fine-scale motions in turbulent channel flow. J. Fluid Mech. 310, 269-292.

Caro, C. G., Fitz-Gerald, J. M. \& Schroter, R. C. 1971 Atheroma and arterial wall shear: observation, correlation and proposal of a shear dependent mass transfer mechanism for atherogenesis. Proc. R. Soc. Lond. B 177, 109-159.

Cassanova, R. A. \& Giddens, D. P. 1978 Disorder distal to modified stenoses in steady and pulsatile flow. J. Biomech. 11, 441-453.

Chomaz, J.-M. 2005 Global instabilities in spatially developing flows: non-normality and nonlinearity. Annu. Rev. Fluid Mech. 37, 357-392.

Chong, M. S., Perry, A. E. \& Cantwell, B. J. 1990 A general classification of three-dimensional flows. Phys. Fluids A 2 (5), 765-777.

Dodds, S. R. \& Phillips, P. S. 2003 The haemodynamics of multiple sequential stenoses and the criteria for a critical stenosis. Eur. J. Endovasc. Surg. 26, 348-353.

Gharib, M., Rambod, E. \& Shariff, K. 1998 A universal time scale for vortex ring formation. J. Fluid Mech. 360, 121-140.

Goldstein, J. A., Demetriou, D., Grines, C., Pica, M. \& O’Neill, W. W. 2000 Multiple complex coronary plaques in patients with acute myocardial infarction. New Engl. J. Med. 343, 915-922.

Guermond, J. \& Shen, J. 2003 Velocity-correction projection methods for incompresible flows. SIAM J. Numer. Anal. 41, 112-134.

Huerre, P. \& Rossi, M. 1998 Hydrodynamic instabilities in open flows. In Hydrodynamics and Nonlinear Instabilities (ed. C. Godrèche \& P. Manneville), pp. 81-294. Cambridge University Press.

Karniadakis, G. E., Israeli, M. \& Orszag, S. A. 1991 High-order splitting methods for the incompressible Navier-Stokes equations. J. Comput. Phys. 97, 414-443.

Khalifa, A. M. A. \& Giddens, D. P. 1981 Characterization and evolution of poststenotic disturbances. J. Biomech. 14, 279-296.

Ku, D. N. 1997 Blood flow in arteries. Annu. Rev. Fluid Mech. 29, 399-434.

Kumaran, V. 1996 Stability of inviscid flow in a flexible tube. J. Fluid Mech. 320, 1-17.

Long, Q., Xu, X. Y., Ramnarine, K. V. \& Hoskins, P. 2001 Numerical investigation of physiologically realistic pulsatile flow through arterial stenosis. J. Biomech. 34, 1229-1242.

McDonald, D. A. 1974 Blood Flow in Arteries, 2nd edn. Edward Arnold.

Mallinger, F. \& Drikakis, D. 2002 Instability in three-dimensional, unsteady, stenotic flows. Intl J. Heat Fluid Flow 23, 657-663.

Mittal, R., Simmons, S. \& NajJar, F. 2003 Numerical study of pulsatile flow in a constricted channel. J. Fluid Mech. 485, 337-378.

Monkewitz, P. A. \& Huerre, P. 1982 Influence of the velocity ratio on the spatial instability of mixing layers. Phys. Fluids 25, 1137-1143.

Nichols, W. W. \& O'Rourke, M. F. 1998 McDonald's Blood Flow in Arteries: Theoretical, Experimental and Clinical Principles, 4th edn. Arnold.

Ojha, M., Cobbold, R. S. C., Johnston, K. W. \& Hummel, R. L. 1989 Pulsatile flow through constricted tubes: an experimental investigation using photochromic tracer methods. J. Fluid Mech. 203, 173-197.

Pedley, T. J. 2000 Blood flow in arteries and veins. In Perspectives in Fluid Dynamics: A Collective Introduction to Current Research (ed. G. K. Batchelor, H. K. Moffatt \& M. G. Worster), chap. 3, pp. 105-158. Cambridge University Press.

Pitt, R., Sherwin, S. J. \& Theofilis, V. 2005 Biglobal stability analysis of steady flow in constricted channel geometries. Intl J. Numer. Meth. Fluids 47, 1227-1235.

ReYnolds, W. C. \& Hussain, A. K. M. F. 1972 The mechanics of an organized wave in turbulent shear flow. Part 3. Theoretical models and comparisons with experiments. J. Fluid Mech. 41 263-288.

Reynolds, W. C., Parekh, D. E., Juvet, P. J. D. \& Lee, M. J. D. 2003 Bifurcating and blooming jets. Annu. Rev. Fluid Mech. 35, 295-315.

Rosenfeld, M., Rambod, E. \& Gharib, M. 1998 Circulation and formation number of laminar vortex rings. J. Fluid Mech. 376, 297-318.

Saffman, P. G. 1992 Vortex Dynamics. Cambridge University Press.

SexL, T. 1930 Über den von E. G. Richardson entdeckten 'annulareffekt'. Z. Phys. 61, 349-362. 
Sherwin, S. J. \& Blackburn, H. M. 2005 Three-dimensional instabilities and transition of steady and pulsatile flows in an axisymmetric stenotic tube. J. Fluid Mech. 533, $297-327$.

Sobey, I. J. \& Drazin, P. G. 1986 Bifurcations of two-dimensional channel flows. J. Fluid Mech. 171, 263-287.

Stroud, J. S., Berger, S. A. \& SAloner, D. 2000 Influence of stenosis morphology on flow through stenotic vessels: implications for plaque rupture. J. Biomech. 33, 443-455.

Stroud, J. S., Berger, S. A. \& Saloner, D. 2002 Numerical analysis of flow through a severely stenotic carotid artery bifurcation. Trans. ASME K: J. Biomech. Engng 124, 9-20.

TAYlor, C. A. \& Draney, M. L. 2004 Experimental and computational methods in cardiovascular fluid mechanics. Annu. Rev. Fluid Mech. 36, 197-231.

Tuckerman, L. S. \& Barkley, D. 2000 Bifurcation analysis for timesteppers. In Numerical Methods for Bifurcation Problems and Large-Scale Dynamical Systems (ed. E. Doedel \& L. S. Tuckerman), pp. 453-566. Springer.

Varghese, S., Frankel, S. \& Fischer, P. 2006 Direct numerical simulation of stenotic flow. Part 2. Pulsatile flow. J. Fluid Mech. (submitted).

Widnall, S. E., Bliss, D. B. \& TsaI, C.-Y. 1974 The instability of short waves on a vortex ring. J. Fluid Mech. 66, 35-47.

Womersley, J. R. 1955 Method for the calculation of velocity, rate of flow and viscous drag in arteries when the pressure gradient is known. J. Physiol. 127, 553-563.

Wootton, D. M. \& Ku, D. N. 1999 Fluid mechanics of vascular systems, diseases, and thrombosis. Annu. Rev. Biomed. Engng 1, 299-329.

YANG, W. H. \& YIH, C.-S. 1977 Stability of time-periodic flows in a circular pipe. J. Fluid Mech. 82, 497-505. 